\title{
A Complete Stability Analysis of Planar Discrete-Time Linear Systems Under Saturation
}

\author{
Tingshu Hu and Zongli Lin, Senior Member, IEEE
}

\begin{abstract}
A complete stability analysis is performed on a planar discrete-time system of the form $x(k+1)=\operatorname{sat}(A x(k))$, where $A$ is a Schur stable matrix and sat is the saturation function. Necessary and sufficient conditions for the system to be globally asymptotically stable are given. In the process of establishing these conditions, the behaviors of the trajectories are examined in detail.
\end{abstract}

Index Terms-Limit trajectories, neural networks, saturation, stability.

\section{INTRODUCTION}

D YNAMICAL systems with saturation nonlinearities arise frequently in neural networks, analogue circuits and control systems (see, for example, [9], [5], [2], [6] and the references therein). In this paper, we consider systems of the following form:

$$
x(k+1)=\operatorname{sat}(A x(k)), \quad x \in \mathbf{R}^{n}
$$

where sat $\mathbf{R}^{n} \rightarrow \mathbf{R}^{n}$ is the standard saturation function. With a slight abuse of notation, we use the same symbol to denote both the vector saturation function and the scalar saturation function, i.e., if $v \in \mathbf{R}^{n}$, then sat $(v)=\left[\operatorname{sat}\left(v_{1}\right) \text {, sat }\left(v_{2}\right), \ldots, \operatorname{sat}\left(v_{n}\right)\right]^{T}$ and

$$
\operatorname{sat}\left(v_{i}\right)= \begin{cases}-1, & \text { if } v_{i}<-1 \\ v_{i}, & \text { if }-1 \leq v_{i} \leq 1 \\ 1, & \text { if } v_{i}>1\end{cases}
$$

Systems of the form (1) and their continuous-time counterparts mainly arise in neural networks and in digital filters.

As with any dynamical system, stability of these systems is of primary concern and has been heavily studied in the literature for a long period of time (see, for example, [1], [7]-[11] and the references therein). As seen in the literature, the stability analysis of such systems are highly nontrivial even for the planar case. For the continuous-time counterpart of (1), only until recently have the necessary and sufficient conditions for global asymptotic stability (GAS) been established for the planar case [4]. For the planar discrete-time system of the form (1), to the best of our knowledge, no necessary and sufficient conditions have been known, although various sufficient conditions are available [9], [11]. This paper attempts to carry out a complete

\footnotetext{
Manuscript received July 6, 2000; revised December 23, 2000. This work was supported in part by the US Office of Naval Research Young Investigator Program under Grant N00014-99-1-0670. This paper was recommended by Associate Editor P. K. Rajan.

The authors are with the Department of Electrical Engineering, University of Virginia, Charlottesville, VA 22903 USA.

Publisher Item Identifier S 1057-7122(01)04290-8.
}

stability analysis of planar systems of the form (1). In particular, necessary and sufficient conditions for the system to be GAS will be identified. In the process of establishing these conditions, the behaviors of the trajectories are examined in detail.

This work is motivated by our recent result [4] on the planar continuous-time system

$$
\dot{x}=\operatorname{sat}(A x), \quad x \in \mathbf{R}^{2} \text {. }
$$

However, the two systems (1) and (3) behave quite differently even though they have a similar description. First of all, (3) operates on the entire plane while (1) operates only on the unit square. The trajectories of (3) do not intersect each other but the connected trajectory of (1) [by connecting $x(k)$ and $x(k+1)$ ] can intersect itself. The limit trajectories of (3) must be periodic but a limit trajectory of (1) need not be. Finally, it is known that in the stability analysis for nonlinear systems, many more tools are available for continuous-time systems than for discrete-time systems.

We will start our investigation of the planar system (1) by characterizing some general properties of its limit trajectories. An important feature is that a nontrivial limit trajectory can only intersect two opposite pair of boundaries of the unit square and it cannot have intersections with both of the neighboring boundaries. This result turns our attention to a simpler system which has only one saturated state

$$
x(k+1)=\left[\begin{array}{c}
a_{11} x_{1}(k)+a_{12} x_{2}(k) \\
\operatorname{sat}\left(a_{21} x_{1}(k)+a_{22} x_{2}(k)\right)
\end{array}\right] .
$$

For this simpler system, we will establish a relation between the present intersection of a trajectory with the lines $x_{2}= \pm 1$ and the next one in terms of a set of points on the line $x_{2}=1$. The relation is discontinuous but piecewise linear. The set of points are the places where the discontinuity occurs. Some attractive properties about these points and the relation between the next intersection and the present one are revealed. These properties help us to establish the condition for the system (4) to be GAS and to characterize an interval on the line $x_{2}=1$ from which the trajectories of (4) will converge to the origin. This in turn leads to our final result on the necessary and sufficient conditions for the GAS of a planar system of the form (1).

This paper is organized as follows. In Section II, we give the necessary and sufficient conditions for the GAS of the planar system in the form of (1). An example is also given to help interpret these conditions. These conditions are established in Sections III-V. In the process of establishing these conditions, the intricate properties of the system trajectories are also revealed. In particular, Section III reveals some general properties of the 
possible limit trajectories of the system which help us to exclude the existence of limit trajectories under the condition of the main theorem and focus our attention to the simpler system with one saturated state. Section IV investigates system (4) and gives a necessary and sufficient condition for the system to be GAS. Section V proves the main result of this paper. Finally, a brief concluding remark is made in Section VI.

\section{MAIN RESUlTS}

Consider the following system:

$$
x(k+1)=\operatorname{sat}(A x(k)), \quad x \in \mathbf{R}^{2}
$$

where $A=\left[\begin{array}{cc}a_{11} & a_{12} \\ a_{21} & a_{22}\end{array}\right]$ and sat: $\mathbf{R}^{2} \rightarrow \mathbf{R}^{2}$ is the saturation function, i.e., if $v \in \mathbf{R}^{2}$, then $\operatorname{sat}(v)=\left[\begin{array}{l}\operatorname{sat}\left(v_{1}\right) \\ \operatorname{sat}\left(v_{2}\right)\end{array}\right]$ and $\operatorname{sat}(\cdot)$ is as defined by (2).

Given an initial state $x(0)=x_{0}$, denote the trajectory of the system (5) that passes through $x_{0}$ at $k=0$ as $\psi\left(k, x_{0}\right)$. In this paper, we only consider the positive trajectories. Hence, throughout the paper, $k \geq 0$.

Definition 2.1: The system (5) is said to be stable at its equilibrium $x_{e}=0$ if, for any $\varepsilon>0$, there exists a $\delta>0$ such that, $\left\|\psi\left(k, x_{0}\right)\right\| \leq \varepsilon$, for all $k \geq 0$ and $\left\|x_{0}\right\| \leq \delta$. It is said to be globally asymptotically stable (GAS) if $x_{e}=0$ is a stable equilibrium and $\lim _{k \rightarrow \infty} \psi\left(k, x_{0}\right)=0$ for all $x_{0} \in \mathbf{R}^{2}$. Also, it is said to be locally asymptotically stable if it is stable and $\lim _{k \rightarrow \infty} \psi\left(k, x_{0}\right)=0$ for all $x_{0}$ in a neighborhood $U_{0}$ of $x_{e}=0$.

The system is GAS only if it is locally asymptotically stable, which is equivalent to that $A$ has eigenvalues inside the unit circle. In this case, $A$ is said to be Schur stable, or simply stable. In this paper, we assume that $A$ is stable. Denote the closed unit square as $\mathbf{S}$ and its boundary as $\partial \mathbf{S}$. It is easy to see that no matter where $x(0)$ is, we always have $x(1) \in \mathbf{S}$. Hence, the global asymptotic stability is equivalent to $\lim _{k \rightarrow \infty} \psi\left(k, x_{0}\right)=$ 0 for all $x_{0} \in \mathbf{S}$. The main result of this paper is presented as follows:

Theorem 2.1: The system (5) is globally asymptotically stable if and only if $A$ is stable and there exists no $x_{0} \in \partial \mathbf{S}$ and $N>0$ such that $\psi\left(N, x_{0}\right)= \pm x_{0}$ and $\psi\left(k, x_{0}\right)=A^{k} x_{0} \in \mathbf{S}$ for all $k<N$.

If $\psi\left(k, x_{0}\right)=A^{k} x_{0} \in \mathbf{S}$ for all $k<N$, then $\psi\left(N, x_{0}\right)=$ $\operatorname{sat}\left(A^{N} x_{0}\right)$. Hence, this theorem can be interpreted as follows. Assume that $A$ is stable, then the system (5) is GAS if and only if none of the following statements are true.

1) There exist $N \geq 1$ and $d_{1}, d_{2} \geq 0$ such that $A^{N}\left[\begin{array}{l}1 \\ 1\end{array}\right]= \pm\left[\begin{array}{l}1+d_{1} \\ 1+d_{2}\end{array}\right] \quad$ and $\quad A^{k}\left[\begin{array}{l}1 \\ 1\end{array}\right] \in \mathbf{S} \quad \forall k<N$

2) There exist $N \geq 1$ and $d_{1}, d_{2} \geq 0$ such that

and

$$
A^{N}\left[\begin{array}{r}
-1 \\
1
\end{array}\right]= \pm\left[\begin{array}{r}
-1-d_{1} \\
1+d_{2}
\end{array}\right]
$$

$$
A^{k}\left[\begin{array}{r}
-1 \\
1
\end{array}\right] \in \mathbf{S} \quad \forall k<N
$$

3) There exist $N \geq 1, d_{2}>0$ and $x_{1} \in(-1,1)$ such that

$$
A^{N}\left[\begin{array}{c}
x_{1} \\
1
\end{array}\right]= \pm\left[\begin{array}{c}
x_{1} \\
1+d_{2}
\end{array}\right]
$$

and

$$
A^{k}\left[\begin{array}{c}
x_{1} \\
1
\end{array}\right] \in \mathbf{S} \quad \forall k<N .
$$

4) There exist $N \geq 1, d_{1}>0$ and $x_{2} \in(-1,1)$ such that

and

$$
A^{N}\left[\begin{array}{c}
1 \\
x_{2}
\end{array}\right]= \pm\left[\begin{array}{c}
1+d_{1} \\
x_{2}
\end{array}\right]
$$

$$
A^{k}\left[\begin{array}{c}
1 \\
x_{2}
\end{array}\right] \in \mathbf{S} \quad \forall k<N
$$

Each of the above conditions implies that there is a simple periodic trajectory that starts at some $x_{0}$ with period $N$ or $2 N$. The trajectory stays inside $\mathbf{S}$ as that of the corresponding linear system for the first $N-1$ steps, and when the linear trajectory goes out of $\mathbf{S}$ at step $N$, the saturation function makes $\psi\left(N, x_{0}\right)=\operatorname{sat}\left(A^{N} x_{0}\right)$ return exactly at $x_{0}$ or $-x_{0}$. These conditions can be verified. Since $A$ is stable, there exists an integer $N_{0}$ such that $A^{k} x_{0} \in \mathbf{S}$ for all $k>N_{0}$ and all $x_{0} \in \partial \mathbf{S}$. Hence, it suffices to check the four conditions only for $N<N_{0}$.

Conditions 1) and 2) are very easy to check. As to 3 ) or 4), for each $N$, at most two $x_{1}^{\prime} s$ can be solved from

$$
A^{N}\left[\begin{array}{c}
x_{1} \\
1
\end{array}\right]= \pm\left[\begin{array}{c}
x_{1} \\
1+d_{2}
\end{array}\right] .
$$

To see this, denote the elements of $A^{N}$ as $\left(A^{N}\right)_{i j}, i, j=1,2$. Then from 3), we have

$$
\left(A^{N}\right)_{11} x_{1}+\left(A^{N}\right)_{12}= \pm x_{1} .
$$

If $\left(A^{N}\right)_{11} \neq \pm 1$, then there are two $x_{1}$ 's that satisfy (6). If $\left(A^{N}\right)_{11}= \pm 1$, we must have $\left(A^{N}\right)_{12} \neq 0$. Otherwise $A^{N}$ would have an eigenvalue \pm 1 , which is impossible since $A$ is stable. In this case, (6) has only one solution. It remains to check if $d_{2}>0$ and

$$
A^{k}\left[\begin{array}{c}
x_{1} \\
1
\end{array}\right] \in \mathbf{S} \quad \forall k<N .
$$

In the process of proving Theorem 2.1, we will develop a more efficient method to check the conditions.

Example 2.1: Consider (5) with

$$
A=\left[\begin{array}{ll}
1.5840 & -1.3990 \\
3.9702 & -2.9038
\end{array}\right] .
$$

The following results are presented with accuracy up to four decimal digits. There are two points on $\partial \mathbf{S}$ that satisfy condition 3 ), one with

$$
x_{1}=\frac{\left(A^{3}\right)_{12}}{1-\left(A^{3}\right)_{11}}=0.7308
$$

and the other with

$$
x_{1}=\frac{\left(A^{2}\right)_{12}}{-1-\left(A^{2}\right)_{11}}=0.9208 .
$$


But there are four periodic trajectories as listed

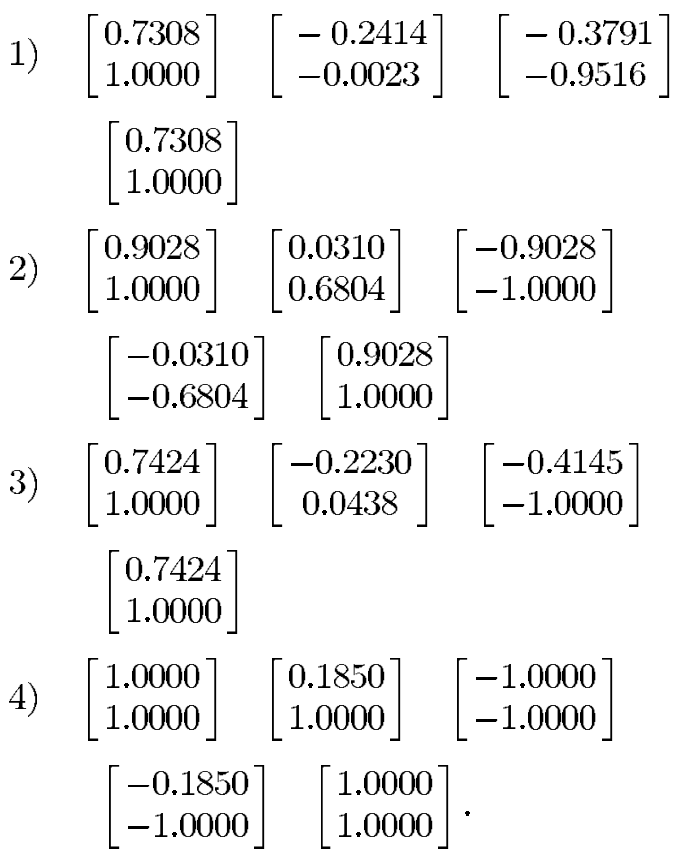

In the third periodic trajectory, the first coordinate $x_{1}=0.7424$ of the initial state is computed from

$$
x_{1}=\frac{a_{12}-a_{11}\left(A^{2}\right)_{12}}{a_{11}\left(A^{2}\right)_{11}-1}=0.7424 .
$$

It should be noted that 4 ) is the only stable periodic trajectory.

As we can see from the example, there are other kinds of periodic trajectories than what are inferred by the conditions 1) -4 ), e.g., trajectories 3) and 4). There may also be trajectories that neither are periodic nor converge to the origin. We will prove in the subsequent sections that if none of the conditions 1)-4) is true, then there exist no nonconvergent trajectory of any kind.

\section{LIMIT TRAJECTORIES}

To prove that (5) is GAS, we need to show that the only limit point of any trajectory is the origin. It is known that $A$ being stable alone is not sufficient to guarantee the GAS of the system. Actually, it is well-known [9] that the system may have stationary points other than the origin; there may be periodic trajectories and even trajectories that neither are periodic, nor converge to a stationary point. In this section, we are going to characterize some general properties of the nonconvergent trajectories. These properties will facilitate us to exclude the existence of such nonconvergent trajectories under the condition of Theorem 2.1.

Since every trajectory is bounded by the unit square, there exists a set of points such that the trajectory will go arbitrarily close to them infinitely many times.

Definition 3.1: For a given $x_{0} \in \mathbf{R}^{2}$, a point $x^{*} \in \mathbf{R}^{2}$ is called a (positive) limit point of the trajectory $\psi\left(k, x_{0}\right)$ if there exists a subsequence of $\psi\left(k, x_{0}\right), \psi\left(k_{i}, x_{0}\right), i=1,2, \ldots$, such that $\lim _{i \rightarrow \infty} \psi\left(k_{i}, x_{0}\right)=x^{*}$. The set of all such limit points is called the limit set of the trajectory. We denote this limit set as $\Gamma\left(x_{0}\right)$.
Since the function $\operatorname{sat}(A x)$ is continuous in $x$, if a trajectory $\psi\left(k, x_{0}\right)$ returns arbitrarily close to $x \in \Gamma\left(x_{0}\right)$, it will also return arbitrarily close to sat $(A x)$. We state this property in the following lemma.

Lemma 3.1: If $y_{0} \in \Gamma\left(x_{0}\right)$, then $\psi\left(k, y_{0}\right) \in \Gamma\left(x_{0}\right)$ for all $k \geq 0$. Given any $\varepsilon>0$ (arbitrarily small) and any integer $N>0$ (arbitrarily large), there exists an integer $K_{0}>0$ such that

$$
\left|\psi\left(k+K_{0}, x_{0}\right)-\psi\left(k, y_{0}\right)\right|_{\infty}<\varepsilon \quad \forall k \leq N .
$$

Because of Lemma 3.1, for $y_{0} \in \Gamma\left(x_{0}\right), \psi\left(k, y_{0}\right)$ is called a limit trajectory of $\psi\left(k, x_{0}\right)$. It is periodic if and only if $\Gamma\left(x_{0}\right)$ has finite number of elements.

The following notation is defined for simplicity. Denote

$$
\begin{aligned}
& L_{h}=\left\{\left[\begin{array}{c}
x_{1} \\
1
\end{array}\right]: x_{1} \in(-1,1)\right\} \\
& L_{v}=\left\{\left[\begin{array}{c}
1 \\
x_{2}
\end{array}\right]: x_{2} \in(-1,1)\right\} .
\end{aligned}
$$

We see that $L_{h}$ and $-L_{h}$ are the two horizontal sides of $\mathbf{S}$, and $L_{v}$ and $-L_{v}$ are the two vertical sides of $\mathbf{S}$. Notice that they do not include the four vertices of the unit square. Also, denote $v_{1}=\left[\begin{array}{l}1 \\ 1\end{array}\right], v_{2}=\left[\begin{array}{c}-1 \\ 1\end{array}\right]$ as the two upper vertices of the square.

Let $y_{0}$ be a limit point of some trajectory and for simplicity, let $y_{k}=\psi\left(k, y_{0}\right)$. Denote $Y=\left\{ \pm y_{k}: k \geq 0\right\}$ and $A Y=$ $\left\{ \pm A y_{k}: k \geq 0\right\}$. Clearly, $Y$ must have an intersection with the boundary of the unit square. If $Y \cap L_{h}$ is not empty, define

$$
\gamma_{1}=\inf \left\{x_{1}:\left[\begin{array}{c}
x_{1} \\
1
\end{array}\right] \in Y \cap\left(L_{h} \cup\left\{v_{1}, v_{2}\right\}\right)\right\}
$$

and

$$
\gamma_{2}=\sup \left\{x_{1}:\left[\begin{array}{c}
x_{1} \\
1
\end{array}\right] \in Y \cap\left(L_{h} \cup\left\{v_{1}, v_{2}\right\}\right)\right\} .
$$

If $Y \cap L_{v}$ is not empty, define

and

$$
\gamma_{3}=\sup \left\{x_{2}:\left[\begin{array}{c}
1 \\
x_{2}
\end{array}\right] \in Y \cap\left(L_{v} \cup\left\{v_{1},-v_{2}\right\}\right)\right\}
$$

$$
\gamma_{4}=\inf \left\{x_{2}:\left[\begin{array}{c}
1 \\
x_{2}
\end{array}\right] \in Y \cap\left(L_{v} \cup\left\{v_{1},-v_{2}\right\}\right)\right\} .
$$

The following proposition shows that a limit trajectory can only intersect one opposite pair of the sides of the unit square, not both of the neighboring pair. This result will reduce our problem to a much simpler one.

Proposition 3.1: Let $y_{0}$ be a limit point of some trajectory.

1) If $y_{0} \in L_{h}$, then $\psi\left(k, y_{0}\right)$ will not touch $L_{v}$ or $-L_{v}$ for all $k \geq 0$. Moreover, $\psi\left(k, y_{0}\right)$ will stay inside the strip

$$
\left\{\left[\begin{array}{l}
x_{1} \\
x_{2}
\end{array}\right]:\left|x_{1}\right| \leq \max \left\{\left|\gamma_{1}\right|,\left|\gamma_{2}\right|\right\}\right\} .
$$

2) If $y_{0} \in L_{v}$, then $\psi\left(k, y_{0}\right)$ will not touch $L_{h}$ or $-L_{h}$ and will stay inside the strip

$$
\left\{\left[\begin{array}{l}
x_{1} \\
x_{2}
\end{array}\right]:\left|x_{2}\right| \leq \max \left\{\left|\gamma_{3}\right|,\left|\gamma_{4}\right|\right\}\right\} .
$$

3) The set $Y$ cannot include both $v_{1}$ and $v_{2}$. 


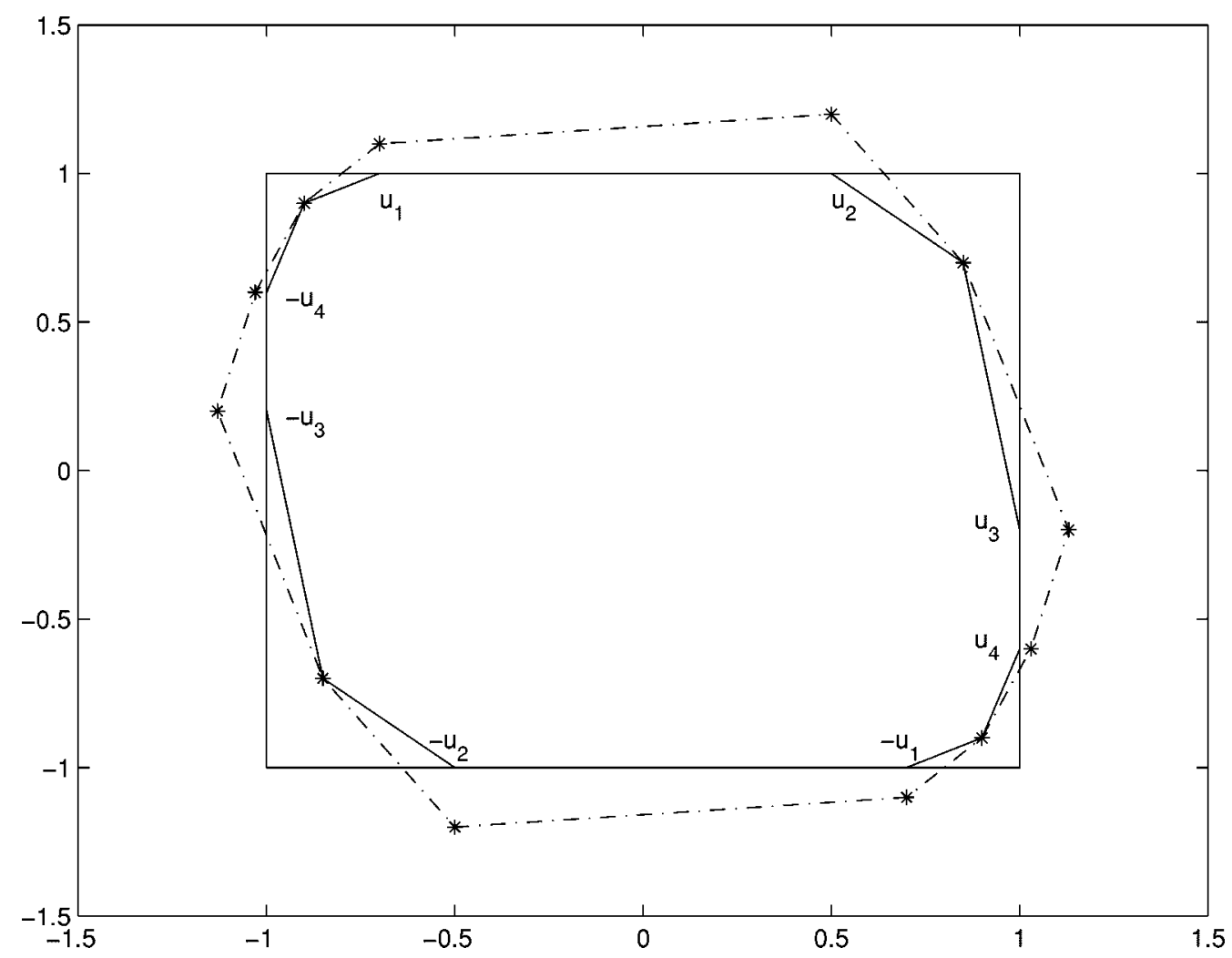

Fig. 1. Illustration for the proof of Proposition 3.1.

Proof: The proof is built up on a simple geometric fact. Let $X$ be a set in $\mathbf{R}^{2}$ and let $A X$ be the image of $X$ under the linear map $x \rightarrow A x$. Then, the area of $A X$ equals to the area of $X$ times $|\operatorname{det}(A)|$.

1)We first assume that $Y$ contains a finite number of elements, i.e., $\psi\left(N, y_{0}\right)=y_{0}$ for some $N$. Suppose on the contrary that the trajectory will touch $L_{v}$ or $-L_{v}$ at some step. The main idea of the proof is to show that the area of the convex hull of $A Y$ is no less than that of $Y$, which contradicts the fact that $|\operatorname{det}(A)|<1$.

Since $Y$ contains points on both $L_{h}$ and $L_{v}, \gamma_{i}$, $i=1,2,3,4$, are all defined.

If $y_{k}$ is in the interior of the unit square, then $y_{k}=A y_{k-1}$; if $y_{k} \in L_{h}$, then $y_{k}=\operatorname{sat}\left(A y_{k-1}\right)$ and

$$
A y_{k-1}=\left[\begin{array}{c}
x_{1} \\
1+d
\end{array}\right]
$$

for some $\left|x_{1}\right|<1$ and $d \geq 0$ [note that $y_{0}=y_{N}=$ $\left.\operatorname{sat}\left(A y_{N-1}\right)\right]$; if $y_{k} \in L_{v}$, then

$$
A y_{k-1}=\left[\begin{array}{c}
1+d \\
x_{2}
\end{array}\right]
$$

for some $\left|x_{2}\right|<1$ and $d \geq 0$. If $y_{k}=v_{1}$ (or $v_{2}$ ), then $y_{k}=$ $\operatorname{sat}\left(A y_{k-1}\right)$ and

$$
A y_{k-1}=\left[\begin{array}{l}
1+d_{1} \\
1+d_{2}
\end{array}\right] \quad\left(\text { or }\left[\begin{array}{c}
-1-d_{1} \\
1+d_{2}
\end{array}\right]\right)
$$

for some $d_{1}, d_{2} \geq 0$. Hence, $A Y$ contains all the elements of $Y$ which are in the interior of $\mathbf{S}$, and for those $y_{k}$ on the boundary of $\mathbf{S}$, if $y_{k} \in L_{h}$, there is a point in $A Y$ that is just above $y_{k}$ (on the same vertical line) and if $y_{k} \in L_{v}$, then there is a point in $A Y$ that is just to the right of $y_{k}$ (on the same horizontal line).

Denote the areas of the convex hulls of $Y$ and $A Y$ as $\mathcal{A}(Y)$ and $\mathcal{A}(A Y)$, respectively. Also, let

$$
\begin{array}{ll}
u_{1}=\left[\begin{array}{c}
\gamma_{1} \\
1
\end{array}\right] & u_{2}=\left[\begin{array}{c}
\gamma_{2} \\
1
\end{array}\right] \\
u_{3}=\left[\begin{array}{c}
1 \\
\gamma_{3}
\end{array}\right] & u_{4}=\left[\begin{array}{c}
1 \\
\gamma_{4}
\end{array}\right]
\end{array}
$$

as shown in Fig. 1. In the figure, the points marked with " $*$ " belong to $A Y$, the polygon with dash-dotted boundary is the convex hull of $A Y$ and the polygon with vertices $\pm u_{i}, i=$ $1,2,3,4$, and some points in the interior of $\mathbf{S}$ is the convex hull of $Y$. Since there is at least one point in $Y$ that is to the left of $u_{1}$, one to the right of $u_{2}$, one above $u_{3}$ and one below $u_{4}$, the convex hull of $Y$ is a subset of the convex hull of $A Y$. (This may not be true if $u_{1}$ is the leftmost point in $Y$, or if $u_{2}$ is the rightmost). It follows that $\mathcal{A}(A Y) \geq \mathcal{A}(Y)$. This is a contradiction since $\mathcal{A}(A Y)=|\operatorname{det}(A)| \mathcal{A}(Y)$ and $|\operatorname{det}(A)|<1$.

If, on the contrary, $Y$ has a point outside of the strip

$$
\left\{\left[\begin{array}{l}
x_{1} \\
x_{2}
\end{array}\right]:\left|x_{1}\right|<\max \left\{\left|\gamma_{1}\right|,\left|\gamma_{2}\right|\right\}\right\}
$$

then, there will be a point in $Y$ that is to the left of $u_{1}$ (or on the same vertical line with $u_{1}$ ), and a point to the right of $u_{2}$ (or on the same horizontal line with $u_{2}$ ). In this case, we also have $\mathcal{A}(A Y) \geq \mathcal{A}(Y)$, which is a contradiction.

Now we extend the result to the case that $Y$ has infinite many elements. Also suppose on the contrary that the trajectory will touch $L_{v},-L_{v}$ or go outside of the strip at some step. By 
Lemma 3.1, for any $\varepsilon>0$ and any integer $N \geq 1$, there exists a $K_{0}>0$ such that

$$
\left|\psi\left(k+K_{0}, x_{0}\right)-\psi\left(k, y_{0}\right)\right|_{\infty}<\varepsilon
$$

for all $k \leq N$ and in particular

$$
\left|\psi\left(K_{0}, x_{0}\right)-y_{0}\right|_{\infty}<\varepsilon
$$

So, the trajectory $\psi\left(k+K_{0}, x_{0}\right), k \geq 0$, will also touch (or almost touch) $L_{v},-L_{v}$, or go outside of the strip. Since $y_{0}$ is a limit point of $\psi\left(k+K_{0}, x_{0}\right)$, there exists a $K_{1}>0$ such that

$$
\left|\psi\left(K_{1}+K_{0}, x_{0}\right)-y_{0}\right|_{\infty}<\varepsilon
$$

Define

$$
Z(\varepsilon)=\left\{\psi\left(k+K_{0}, x_{0}\right): 0 \leq k \leq K_{1}\right\}
$$

and

$$
A Z(\varepsilon)=\left\{A \psi\left(k+K_{0}, x_{0}\right): 0 \leq k \leq K_{1}\right\} .
$$

Using similar arguments as in the finite element case, we can show that

$$
|\operatorname{det}(A)|=\frac{\mathcal{A}(A Z(\varepsilon))}{\mathcal{A}(Z(\varepsilon))} \geq 1-O(\varepsilon) .
$$

Letting $\varepsilon \rightarrow 0$, we obtain $|\operatorname{det}(A)| \geq 1$, which is a contradiction.

2) Similar to 1$)$.

3) If, on the contrary, $Y$ contains both $v_{1}$ and $v_{2}$, then the convex hull of $Y$ is $\mathbf{S}$. Also, $A Y$ contains a point

$$
A y_{j}=\left[\begin{array}{l}
x_{1} \\
x_{2}
\end{array}\right], \quad x_{1} \leq-1 \quad x_{2} \geq 1
$$

and a point

$$
A y_{k}=\left[\begin{array}{l}
x_{1} \\
x_{2}
\end{array}\right], \quad x_{1} \geq 1 \quad x_{2} \geq 1
$$

hence, the convex hull of $A Y$ contains $\mathbf{S}$. This also leads to $\mathcal{A}(A Y) \geq \mathcal{A}(Y)$, a contradiction.

\section{Systems with One Saturated State}

Now, we are clear from Proposition 3.1 that if there is any limit trajectory, it can intersect only one opposite pair of the sides of the unit square, either $\left(L_{h},-L_{h}\right)$, or $\left(L_{v},-L_{v}\right)$, not both of them. So, we only need to investigate the possibility that a limit trajectory only intersects $\pm L_{h}$. The other possibility that it only intersects $\pm L_{v}$ is similar. For this reason, we consider the following system:

$$
x(k+1)=\left[\begin{array}{c}
a_{11} x_{1}(k)+a_{12} x_{2}(k) \\
\operatorname{sat}\left(a_{21} x_{1}(k)+a_{22} x_{2}(k)\right)
\end{array}\right]:=\operatorname{sat}_{2}(A x(k)) .
$$

Assume that $A=\left[\begin{array}{ll}a_{11} & a_{12} \\ a_{21} & a_{22}\end{array}\right]$ is stable. If $a_{21}=0$ or $a_{12}=0$, it is easy to see that both systems (5) and (7) are GAS and none of the conditions 1)-4) following Theorem 2.1 can be true. So we assume in the following that $a_{21}, a_{12} \neq 0$.
The terms GAS, limit point and limit trajectory for (5) are extended to (7) in a natural way.

For a given initial state $x(0)=x_{0}$, denote the trajectory of the system (7) as $\psi_{2}\left(k, x_{0}\right)$. Denote the line $x_{2}=1$ as $L_{h}^{e}$, the line $x_{2}=-1$ as $-L_{h}^{e}$ and the region between these two lines (including $\pm L_{h}^{e}$ ) as $\mathbf{S}^{e}$. We will show later that (7) has nontrivial limit trajectory in $\mathbf{S}$ if and only if (5) has nontrivial limit trajectory that intersects $\pm L_{h}$. In the sequel, when we say "limit trajectory," we mean a limit trajectory other than the trivial one at the origin.

In this section, we study the GAS of the system (7) and will also determine a subset in $L_{h}^{e}$ which is free of limit points. Our investigation will be based on the study of the linear system

$$
x(k+1)=A x(k) .
$$

For a stable continuous-time linear planar system, if a trajectory stays in $\mathbf{S}^{e}$ for a whole cycle [ $\angle x(t)$ increases or decreases by $2 \pi$, then $x(t)$ will be in $\mathbf{S}^{e}$ for all $t>0$. But, for the discrete-time linear planar system (8), a trajectory might go out of $\mathbf{S}^{e}$ after staying within $\mathbf{S}^{e}$ for several cycles. In the continuous-time case, the trajectories never intersect but in the discrete-time case, the connected trajectory [by connecting $x(k)$ and $x(k+1)$ ] can intersect itself. These facts make the analysis much more complicated than the continuous-time system as discussed in [1], [3], [4] and [10].

A simple one or two point periodic trajectory can be formed if $A\left[\begin{array}{c}x_{1} \\ 1\end{array}\right]= \pm\left[\begin{array}{c}x_{1} \\ 1+d\end{array}\right]$ for some $d>0$. An $N$ or $2 N$ point periodic trajectory will be formed if $A^{N}\left[\begin{array}{c}x_{1} \\ 1\end{array}\right]= \pm\left[\begin{array}{c}x_{1} \\ 1+d\end{array}\right], d>0$ and $A^{k}\left[\begin{array}{c}x_{1} \\ 1\end{array}\right] \in \mathbf{S}^{e}$, for all $k<N$.

Proposition 4.1: The system (7) is GAS if and only if $A$ is stable and the following statement is not true for any $x_{1} \in \mathbf{R}$ : There exist an integer $N>0$ and a real number $d>0$ such that

and

$$
A^{N}\left[\begin{array}{c}
x_{1} \\
1
\end{array}\right]= \pm\left[\begin{array}{c}
x_{1} \\
1+d
\end{array}\right]
$$

$$
A^{k}\left[\begin{array}{c}
x_{1} \\
1
\end{array}\right] \in \mathbf{S}^{e} \quad \forall k<N .
$$

Let $\alpha_{s}=\min \left\{\left|x_{1}\right|: x_{1}\right.$ satisfies (9) $\}$, then no limit trajectory can exist completely within the strip

$$
\left\{\left[\begin{array}{l}
x_{1} \\
x_{2}
\end{array}\right]:\left|x_{1}\right|<\alpha_{s}\right\}
$$

Remark: If (9) is true for some $x_{1}$, then there will be a stationary point or periodic trajectory such as $x_{0}, A x_{0}, \ldots, A^{N-1} x_{0}, \operatorname{sat}_{2}\left(A^{N} x_{0}\right)=x_{0}, A x_{0}, \ldots$. There may also exist other kind of limit trajectories. Proposition 4.1 says that if there is no simple periodic trajectory as inferred by (9), there will be no limit trajectory of any kind (except the one at the origin).

To prove Proposition 4.1, we need to establish the relation between the next intersection of a trajectory with $\pm L_{h}^{e}$ and the present one.

For $x_{0} \in L_{h}^{e}$, suppose that $\psi_{2}\left(k, x_{0}\right)$ will intersect $\pm L_{h}^{e}$ at $k=k_{i}, i=1,2, \ldots$, with $0<k_{1}<k_{2}<\ldots$. Since the trajectory can be switched to $-\psi_{2}\left(k, x_{0}\right)$ at any $k$ without changing 
its convergence property, we assume for simplicity that all the intersections $\psi_{2}\left(k_{i}, x_{0}\right)$ are in $L_{h}^{e}$ (If not so, just multiply it with $-1)$. Denote

$$
x_{0}^{1}=\psi_{2}\left(k_{1}, x_{0}\right) \quad x_{0}^{2}=\psi_{2}\left(k_{2}, x_{0}\right) \quad \ldots
$$

We call $x_{0}, x_{0}^{1}$ and $x_{0}^{2}$ the first, the second and the third intersections, respectively. We also call $x_{0}$ and $x_{0}^{1}$ the present and the next intersections.

Clearly, $x_{0}^{1}$ is uniquely determined by $x_{0}$. We also see that the relation $x_{0} \rightarrow x_{0}^{1}$ is a map from $L_{h}^{e}$ to itself. To study the GAS of the system (7), it suffices to characterize the relation between $x_{0}$ and $x_{0}^{1}$. Through this relation, we can show that if (9) is not true for any $x_{1}$, then for every $x_{0} \in L_{h}^{e}$, the intersections $x_{0}^{1}$, $x_{0}^{2}, \ldots$ will move closer and closer toward an interval, and all the trajectories starting from this interval will not touch the lines $\pm L_{h}^{e}$ and will converge to the origin.

Let $x_{0} \in L_{h}^{e}$. The next intersection of $\psi_{2}\left(k, x_{0}\right)$ with $L_{h}^{e}$ occurs at step $k_{1}$ if

and

$$
\left|\left[\begin{array}{ll}
0 & 1
\end{array}\right] A^{k_{1}} x_{0}\right| \geq 1
$$

$$
\left|\left[\begin{array}{ll}
0 & 1
\end{array}\right] A^{k} x_{0}\right|<1 \quad \forall k<k_{1} .
$$

The next intersection is $x_{0}^{1}=\psi_{2}\left(k_{1}, x_{0}\right)=\operatorname{sat}_{2}\left(A^{k_{1}} x_{0}\right)$ [or - $\left.\operatorname{sat}_{2}\left(A^{k_{1}} x_{0}\right)\right]$. Since for different $x_{0} \in L_{h}^{e}$, the number of steps for the trajectories to return to $\pm L_{h}^{e}$, i.e., the number $k_{1}$ as defined above, is different, we see that the relation between $x_{0}$ and $x_{0}^{1}$ must be discontinuous.

We will first determine an interval on $L_{h}^{e}$ from which a trajectory will not intersect $\pm L_{h}^{e}$ again (no $x_{0}^{1}$ ) and will converge to the origin.

Since $A$ is stable, there exists a positive definite matrix $P$ such that

$$
A^{T} P A-P<0 \text {. }
$$

Define the Lyapunov function as

$$
V(x):=x^{T} P x
$$

then for every $x \in \mathbf{R}^{2}, V\left(A^{k} x\right)<V(x)$ for all $k>1$.

Given a real number $\rho>0$, denote the Lyapunov level set as

$$
\mathcal{E}(\rho):=\left\{x \in \mathbf{R}^{2}: x^{T} P x \leq \rho\right\} .
$$

Let $\rho_{c}$ be such that $\mathcal{E}\left(\rho_{c}\right) \subset \mathbf{S}^{e}$ and $\mathcal{E}\left(\rho_{c}\right)$ just touches $\pm L_{h}^{e}$. In this case, $\mathcal{E}\left(\rho_{c}\right)$ has only one intersection with $L_{h}^{e}$. Let this intersection be

$$
p_{c}=\left[\begin{array}{c}
\alpha_{c} \\
1
\end{array}\right]
$$

If $x_{0}=p_{c}$, then the linear trajectory $A^{k} x_{0}$ will be inside $\mathcal{E}\left(\rho_{c}\right) \subset \mathbf{S}^{e}$. Hence, $\psi_{2}\left(k, x_{0}\right)=\operatorname{sat}_{2}\left(A^{k} x_{0}\right)=A^{k} x_{0}$ for all $k>0$ and will converge to the origin. Since

$$
A^{T} P A-P<0
$$

there exists an interval around $p_{c}$ in $L_{h}^{e}$, of nonzero length, such that for every $x_{0}$ in this interval, $\psi_{2}\left(k, x_{0}\right)=A^{k} x_{0}, k \geq 1$, will never touch $\pm L_{h}^{e}$ and will converge to the origin.
Here we will use a simple way to denote a line segment. Given two points $p_{1}, p_{2} \in \mathbf{R}^{2}$, denote

$$
\left[p_{1}, p_{2}\right]:=\left\{\lambda p_{1}+(1-\lambda) p_{2}: 0 \leq \lambda \leq 1\right\}
$$

and similarly

$$
\begin{aligned}
\left(p_{1}, p_{2}\right] & =\left[p_{1}, p_{2}\right] \backslash\left\{p_{1}\right\} \\
{\left[p_{1}, p_{2}\right) } & =\left[p_{1}, p_{2}\right] \backslash\left\{p_{2}\right\} \\
\left(p_{1}, p_{2}\right) & =\left[p_{1}, p_{2}\right] \backslash\left\{p_{1}, p_{2}\right\} .
\end{aligned}
$$

Define

and

$$
\alpha_{0}:=\min \left\{\alpha<\alpha_{c}: A^{k}\left[\begin{array}{l}
\alpha \\
1
\end{array}\right] \in \mathbf{S}^{e} \quad \forall k \geq 0\right\}
$$

$$
\beta_{0}:=\max \left\{\beta>\alpha_{c}: A^{k}\left[\begin{array}{l}
\beta \\
1
\end{array}\right] \in \mathbf{S}^{e} \quad \forall k \geq 0\right\} .
$$

Since $a_{21} \neq 0$, the line $A L_{h}^{e}:=\left\{A x: x \in L_{h}^{e}\right\}$ has intersections with both $L_{h}^{e}$ and $-L_{h}^{e}$, so there exist points on both sides of $p_{c}$ which will be mapped out of $\mathbf{S}^{e}$ under $A$. Hence $\alpha_{0}$ and $\beta_{0}$ are finite numbers. Now, let

$$
p_{0}=\left[\begin{array}{c}
\alpha_{0} \\
1
\end{array}\right] \quad q_{0}=\left[\begin{array}{c}
\beta_{0} \\
1
\end{array}\right]
$$

then, for all $x_{0} \in\left[p_{0}, q_{0}\right], \psi_{2}\left(k, x_{0}\right)=A^{k} x_{0}$ will converge to the origin. Because of the extremal nature in the definition of $\alpha_{0}$ and $\beta_{0}$, we must have $A^{k} p_{0} \in \pm L_{h}^{e}$ for some $k$, otherwise $\alpha_{0}$ would not be the minimum of the set. Therefore, define

$$
m_{0}:=\min \left\{k: A^{k} p_{0} \in \pm L_{h}^{e}\right\}
$$

and similarly

$$
n_{0}:=\min \left\{k: A^{k} q_{0} \in \pm L_{h}^{e}\right\}
$$

If $m_{0}>1$, then by definition

$$
\left|\left[\begin{array}{ll}
0 & 1
\end{array}\right] A^{k} p_{0}\right|<1 \quad \forall k<m_{0}
$$

and by continuity, there exists a neighborhood of $p_{0}$ such that $\left|\left[\begin{array}{ll}0 & 1\end{array}\right] A^{k} x_{0}\right|<1, \forall k<m_{0}$ for all $x_{0}$ in this neighborhood. Because of this, we can define

$$
\alpha_{1}:=\min \left\{\alpha<\alpha_{0}: A^{k}\left[\begin{array}{l}
\alpha \\
1
\end{array}\right] \in \mathbf{S}^{e} \quad \forall k<m_{0}\right\} .
$$

If $n_{0}>1$, then define

$$
\beta_{1}:=\max \left\{\beta>\beta_{0}: A^{k}\left[\begin{array}{c}
\beta \\
1
\end{array}\right] \in \mathbf{S}^{e}, \quad \forall k<n_{0}\right\} .
$$

Also, because $a_{21} \neq 0, \alpha_{1}$ and $\beta_{1}$ are finite. Let

$$
p_{1}=\left[\begin{array}{c}
\alpha_{1} \\
1
\end{array}\right] \quad q_{1}=\left[\begin{array}{c}
\beta_{1} \\
1
\end{array}\right]
$$

It follows from the extremal nature in the definition of $\alpha_{1}$ and $\beta_{1}$ that there exists a $k<m_{0}$ such that $A^{k} p_{1} \in \pm L_{h}^{e}$, so we define

$$
m_{1}:=\min \left\{k: A^{k} p_{1} \in \pm L_{h}^{e}\right\}
$$


and similarly

$$
n_{1}:=\min \left\{k: A^{k} q_{1} \in \pm L_{h}^{e}\right\} .
$$

For simplicity, we denote $\left[\begin{array}{c}-\infty \\ 1\end{array}\right]$ as $p_{\infty}$ and $\left[\begin{array}{c}\infty \\ 1\end{array}\right]$ as $q_{\infty}$. Implied by the definitions are the following:

$$
p_{1} \in\left(p_{\infty}, p_{0}\right) \quad q_{1} \in\left(q_{0}, q_{\infty}\right),
$$

and

$$
m_{1}<m_{0} \quad n_{1}<n_{0} .
$$

Inductively, if $m_{i-1}>1$, then define

$$
\alpha_{i}:=\min \left\{\alpha<\alpha_{i-1}: A^{k}\left[\begin{array}{l}
\alpha \\
1
\end{array}\right] \in \mathbf{S}^{e} \quad \forall k<m_{i-1}\right\}
$$

and if $n_{j-1}>1$, define

$$
\beta_{j}:=\max \left\{\beta>\beta_{j-1}: A^{k}\left[\begin{array}{c}
\beta \\
1
\end{array}\right] \in \mathbf{S}^{e} \quad \forall k<n_{j-1}\right\} .
$$

Let

$$
p_{i}=\left[\begin{array}{c}
\alpha_{i} \\
1
\end{array}\right] \quad q_{j}=\left[\begin{array}{c}
\beta_{j} \\
1
\end{array}\right]
$$

and

$m_{i}:=\min \left\{k: A^{k} p_{i} \in \pm L_{h}^{e}\right\} \quad n_{j}:=\min \left\{k: A^{k} q_{j} \in \pm L_{h}^{e}\right\}$.

Then

$$
\begin{aligned}
p_{i} & \in\left(p_{\infty}, p_{i-1}\right) \quad q_{j} \in\left(q_{j-1}, q_{\infty}\right), \\
m_{i} & <m_{i-1} \quad n_{j}<n_{j-1} .
\end{aligned}
$$

The induction procedure ends if both $m_{i}=1$ and $n_{j}=1$. We denote the maximum index of $i$ as $I$ and the maximum index of $j$ as $J$. As an immediate consequence of these definitions, we have

$$
\begin{aligned}
\alpha_{I} & <\alpha_{I-1}<\cdots<\alpha_{1}<\alpha_{0}<\alpha_{c}<\beta_{0}<\beta_{1}<\cdots \\
& <\beta_{J-1}<\beta_{J}
\end{aligned}
$$

and

$$
\begin{aligned}
& 1=m_{I}<m_{I-1}<\cdots<m_{1}<m_{0} \\
& 1=n_{J}<n_{J-1}<\cdots<n_{1}<n_{0} .
\end{aligned}
$$

We claim that this set of $p_{i}, i=0,1,2, \ldots, I$, and $q_{j}, j=$ $0,1, \ldots, J$, forms exactly the set of points where discontinuity occurs on the relation between the next intersection of a trajectory with $\pm L_{h}^{e}$ and the present one.

\section{Lemma 4.1:}

1) If $x_{0} \in\left[p_{0}, q_{0}\right]$, then $\psi_{2}\left(k, x_{0}\right)=A^{k} x_{0}$ will be inside $\mathbf{S}^{e}$ for all $k>0$ and will converge to the origin.

2) If $x_{0} \in\left(p_{i+1}, p_{i}\right]$, then the next intersection of $\psi_{2}\left(k, x_{0}\right)$ with $\pm L_{h}^{e}$ is $\psi_{2}\left(m_{i}, x_{0}\right)=\operatorname{sat}_{2}\left(A^{m_{i}} x_{0}\right)$; If $x_{0} \in\left(p_{\infty}, p_{I}\right]$, then the next intersection is $\psi_{2}\left(1, x_{0}\right)=\operatorname{sat}_{2}\left(A x_{0}\right)$. Moreover, $A^{m_{i}} p_{i} \in \pm L_{h}^{e}$ and $A^{m_{i}} x_{0} \notin \mathbf{S}^{e}$ for all $x_{0} \in\left(p_{\infty}, p_{i}\right)$.

3) If $x_{0} \in\left[q_{j}, q_{j+1}\right)$, then the next intersection of $\psi_{2}\left(k, x_{0}\right)$ with $\pm L_{h}^{e}$ is $\psi_{2}\left(n_{j}, x_{0}\right)=\operatorname{sat}_{2}\left(A^{n_{j}} x_{0}\right)$; If $x_{0} \in\left[q_{J}, q_{\infty}\right)$, then the next intersection is $\psi_{2}\left(1, x_{0}\right)=\operatorname{sat}_{2}\left(A x_{0}\right)$. Moreover, $A^{n_{j}} q_{j} \in \pm L_{h}^{e}$ and $A^{n_{j}} x_{0} \notin \mathbf{S}^{e}$ for all $x_{0} \in\left(q_{j}, q_{\infty}\right)$.
4) $\left|\left[\begin{array}{ll}0 & 1\end{array}\right] A^{k} p_{i+1}\right| \leq 1$ for all $k<m_{i}$ and $\left|\left[\begin{array}{ll}0 & 1\end{array}\right] A^{m_{i}} p_{i+1}\right|>$ $1 ;\left|\left[\begin{array}{ll}0 & 1\end{array}\right] A^{k} q_{j+1}\right| \leq 1$ for all $k<n_{j}$ and $\left|\left[\begin{array}{ll}0 & 1\end{array}\right] A^{n_{j}} q_{j+1}\right|>$ 1.

Proof: 1) This is a direct consequence of the definition of $p_{0}$ and $q_{0}$.

2) From the definition of $m_{i},\left|\left[\begin{array}{ll}0 & 1\end{array}\right] A^{k} p_{i}\right|<1$ for all $k<$ $m_{i}$ and $\left|\left[\begin{array}{ll}0 & 1\end{array}\right] A^{m_{i}} p_{i}\right|=1$. Since $A^{m_{i}} L_{h}^{e}$ is a straight line and $A^{m_{i}} p_{c}$ is in the interior of $\mathbf{S}^{e}$, we have $\left|\left[\begin{array}{ll}0 & 1\end{array}\right] A^{m_{i}} x_{0}\right|>1$ for all $x_{0} \in\left(p_{\infty}, p_{i}\right)$ (Note that $p_{c}$ is to the right of $p_{i}$ ).

On the other hand, since

$$
\alpha_{i+1}=\min \left\{\alpha<\alpha_{i}: A^{k}\left[\begin{array}{l}
\alpha \\
1
\end{array}\right] \in \mathbf{S}^{e} \quad \forall k<m_{i}\right\}
$$

we have $\left|\left[\begin{array}{ll}0 & 1\end{array}\right] A^{k} p_{i+1}\right| \leq 1$ for all $k<m_{i}$. Also since $A^{k} p_{c}$ is in the interior of $\mathbf{S}^{e}$, we have $\left|\left[\begin{array}{ll}0 & 1\end{array}\right] A^{k} x_{0}\right|<1$ for all $k<m_{i}$ and for all $x_{0} \in\left(p_{i+1}, p_{c}\right)$.

Combining the above arguments we have, for all $x_{0} \in\left(p_{i+1}, p_{i}\right],\left|\left[\begin{array}{ll}0 & 1\end{array}\right] A^{m_{i}} x_{0}\right| \geq 1$ and $\left|\left[\begin{array}{ll}0 & 1\end{array}\right] A^{k} x_{0}\right|<1$ for all $k<m_{i}$. This means that the next intersection with $\pm L_{h}^{e}$ is $\psi_{2}\left(m_{i}, x_{0}\right)=\operatorname{sat}_{2}\left(A^{m_{i}} x_{0}\right)$.

3) Similar to 2).

4) This is contained in the proof of 2).

It is obvious that $\operatorname{sat}_{2}\left(A^{m_{i}} x\right)$ is a continuous function of $x$. Lemma 4.12$)$ implies that for all $x_{0} \in\left(p_{i+1}, p_{i}\right]$, the second coordinate of $\operatorname{sat}_{2}\left(A^{m_{i}} x_{0}\right),[01] \operatorname{sat}_{2}\left(A^{m_{i}} x_{0}\right)$, is the constant 1 or -1 , while the first coordinate remains linear on $x_{0}$. Similarly, for all $x_{0} \in\left[q_{j}, q_{j+1}\right)$, the second coordinate of $\operatorname{sat}_{2}\left(A^{n_{j}} x_{0}\right)$ is the constant 1 or -1 and the first coordinate is linear on $x_{0}$. Same relation holds for $x_{0} \in\left(p_{\infty}, p_{I}\right]$ and $x_{0} \in\left[q_{I}, q_{\infty}\right)$.

We will provide an easy way to compute $p_{i}$ and $q_{j}$ after revealing more properties about this set of points. In fact, the following properties will lead directly to the proof of Proposition 4.1. For $x_{0} \in L_{h}^{e}$, the next intersection of $\psi_{2}\left(k, x_{0}\right)$ with $\pm L_{h}^{e}$ can be on $L_{h}^{e}$ or on $-L_{h}^{e}$. For simplicity, we will assume that the next intersection is on $L_{h}^{e}$, otherwise we can replace the state $x(k)$ at the intersection with $-x(k)$, noting that we can multiply the state at any step with -1 without changing the convergence rate of a trajectory. Hence in the following, when we say that $x \in\left[p_{i}, q_{j}\right]$, we mean $x \in \pm\left[p_{i}, q_{j}\right]$; and when we say that $x \in \pm L_{h}^{e}$ is to the left (or right) of $p_{i}$, it could also be that $x$ is to the right (or left) of $-p_{i}$.

Denote $p_{i}^{1}=\psi_{2}\left(m_{i}, p_{i}\right)=A^{m_{i}} p_{i}$ and $q_{j}^{1}=\psi_{2}\left(n_{j}, q_{j}\right)=$ $A^{n_{j}} q_{j}$. We see that $p_{i}^{1}$ is the second intersection of $\psi_{2}\left(k, p_{i}\right)$ with $\pm L_{h}^{e}$ (the first one is $p_{i}$ ), and $q_{j}^{1}$ is the second intersection of $\psi_{2}\left(k, q_{j}\right)$ with $\pm L_{h}^{e}$.

\section{Lemma 4.2:}

1) $p_{0}^{1}, q_{0}^{1} \in\left[p_{0}, q_{0}\right]$;

2) If $p_{i}^{1} \in\left(q_{j-1}, q_{j}\right]$, then $m_{i-1}=m_{i}+n_{j-1}$; if $q_{j}^{1} \in$ $\left[p_{i}, p_{i-1}\right)$, then $n_{j-1}=n_{j}+m_{i-1}$

3) For $i \geq 1, p_{i}^{1} \in\left(q_{0}, q_{\infty}\right)$ and for $j \geq 1, q_{j}^{1} \in\left(p_{\infty}, p_{0}\right)$;

4) $p_{i}^{1} \in\left(p_{i-1}^{1}, q_{\infty}\right)$, and $q_{j}^{1} \in\left(p_{\infty}, q_{j-1}^{1}\right)$;

5) For $i, j \geq 1, p_{i}^{1}$ and $q_{j}^{1}$ cannot be both in $\left[p_{i}, q_{j}\right]$, nor both outside of $\left[p_{i}, q_{j}\right]$, i.e., there must be one of them inside $\left[p_{i}, q_{j}\right]$ and the other one outside of the interval.

Proof: First, we give a simple property arising from the Lyapunov function $V(x)$. Since $V(x)$ is a convex function and $p_{c}$ takes the minimum value from all $x \in L_{h}^{e}$, we have, if both 
$s_{1}$ and $s_{2}$ are to the left of $p_{c}$ and $s_{1}$ is to the left of $s_{2}$, then $V\left(s_{1}\right)>V\left(s_{2}\right)$; if both $s_{1}$ and $s_{2}$ are to the right of $p_{c}$ and $s_{1}$ is to the right of $s_{2}$, then $V\left(s_{1}\right)>V\left(s_{2}\right)$.

1)Clearly, $p_{0}^{1}$ cannot be to the left of $p_{0}$, otherwise we would have $V\left(p_{0}^{1}\right)=V\left(A^{n_{0}} p_{0}\right)>V\left(p_{0}\right)$. Suppose on the contrary that $p_{0}^{1} \in\left(q_{0}, q_{\infty}\right)$, then by Lemma 4.13$)$, we would have $\left|\left[\begin{array}{ll}0 & 1\end{array}\right] A^{n_{0}} A^{m_{0}} p_{0}\right|>1$. A contradiction to the definition of $p_{0}$. Similar argument holds for $q_{0}$.

2) From Lemma 4.14), the first time $A^{k} p_{i}$ goes out of $\mathbf{S}^{e}$ is at $k=m_{i-1}$. And by Lemma 4.13$)$ and 4), for $x_{0} \in\left(q_{j-1}, q_{j}\right]$, the first time $A^{k} x_{0}$ goes out of $\mathbf{S}^{e}$ is $k=n_{j-1}$. Since $p_{i}^{1}=$ $A^{m_{i}} p_{i} \in\left(q_{j-1}, q_{j}\right]$ and $A^{k} p_{i} \in \mathbf{S}^{e}$ for all $k<m_{i}$, we have $m_{i-1}=m_{i}+n_{j-1}$. Similarly, for $q_{j}$, we have $n_{j-1}=n_{j}+$ $m_{i-1}$.

3) Similar to 1), $p_{i}^{1}$ cannot be to the left of $p_{i}$. Suppose on the contrary, that $p_{i}^{1} \in\left[p_{0}, q_{0}\right]$, then $A^{k} p_{i}$ never goes out of $\mathbf{S}^{e}$. This is a contradiction since $A^{n_{0}} p_{i} \notin \mathbf{S}^{e}$. Also, suppose on the contrary that $p_{i}^{1} \in\left[p_{i-l}, p_{i-l-1}\right), l \geq 0$, then similar to the argument in 2), we would have $m_{i-1}=m_{i}+m_{i-l-1}$ and hence $m_{i-1}>m_{i-l-1}$. This is a contradiction since $m_{i}$ decreases as $i$ is increased. So, we must have $p_{i}^{1} \in\left(q_{0}, q_{\infty}\right)$ and similarly, $q_{j}^{1} \in\left(p_{\infty}, p_{0}\right)$.

4) Since $p_{i}$ is to the left of $p_{i-1}$, we have

$$
V\left(p_{i}\right)>V\left(p_{i-1}\right)>V\left(p_{c}\right) \text {. }
$$

By 3), $p_{i}^{1}$ is to the right of $p_{c}$, this implies

$$
V\left(p_{c}\right)<V\left(p_{i}^{1}\right)=V\left(A^{m_{i}} p_{i}\right)
$$

and hence

$$
V\left(A^{m_{i}} p_{c}\right)<V\left(p_{c}\right)<V\left(A^{m_{i}} p_{i}\right) .
$$

Since $p_{i-1} \in\left(p_{i}, p_{c}\right)$, the point $A^{m_{i}} p_{i-1}$ is on the line between $A^{m_{i}} p_{c}$ and $A^{m_{i}} p_{i}$. Also, since the function $V(x)$ is convex, it follows from (10) that

$$
V\left(A^{m_{i}} p_{i-1}\right)<\max \left\{V\left(A^{m_{i}} p_{c}\right), V\left(A^{m_{i}} p_{i}\right)\right\}=V\left(A^{m_{i}} p_{i}\right) .
$$

Since $m_{i-1}>m_{i}$, we have $V\left(A^{m_{i-1}} p_{i-1}\right)<V\left(A^{m_{i}} p_{i-1}\right)$ and

$$
\begin{aligned}
V\left(p_{i-1}^{1}\right) & =V\left(A^{m_{i-1}} p_{i-1}\right)<V\left(A^{m_{i}} p_{i-1}\right) \\
& <V\left(A^{m_{i}} p_{i}\right)=V\left(p_{i}^{1}\right) .
\end{aligned}
$$

By 3), $p_{i-1}^{1}$ and $p_{i}^{1}$ are both to the right of $p_{c}$, hence the inequality (11) implies that $p_{i}^{1}$ is to the right of $p_{i-1}^{1}$, i.e., $p_{i}^{1} \in$ $\left(p_{i-1}^{1}, q_{\infty}\right)$.

Similarly, we have $q_{j}^{1} \in\left(p_{\infty}, q_{j-1}^{1}\right)$. have

5) Suppose that both $q_{j}^{1}, p_{i}^{1} \in\left[p_{i}, q_{j}\right]$, then by 2) and 3), we

$$
n_{j-1}=n_{j}+m_{i-k: 1} \quad m_{i-1}=m_{i}+n_{j-k 2}
$$

where $k_{1}, k_{2} \geq 1$. Since $m_{i-1} \leq m_{i-k 1}$ and $n_{j-1} \leq n_{j-k 2}$, it follows that

$$
m_{i-1}<n_{j-1} \quad n_{j-1}<m_{i-1}
$$

which is a contradiction.

On the other hand, suppose that both $q_{j}^{1}, p_{i}^{1} \notin\left[p_{i}, q_{j}\right]$, then we must have $q_{j}^{1}$ to the left of $p_{i}$ and $p_{i}^{1}$ to the right of $q_{j}$. Hence

$$
V\left(q_{j}^{1}\right)>V\left(p_{i}\right) \quad V\left(p_{i}^{1}\right)>V\left(q_{j}\right) .
$$

Recall that $q_{j}^{1}=A^{n_{j}} q_{j}$ and $p_{i}^{1}=A^{m_{i}} p_{i}$, it follows that

$$
V\left(q_{j}^{1}\right)>V\left(p_{i}\right)>V\left(p_{i}^{1}\right)>V\left(q_{j}\right)>V\left(q_{j}^{1}\right)
$$

which is also a contradiction.

From 1), 3), and 5) of Lemma 4.2, we can see that the only pair of $p_{i}$ and $q_{j}$ such that $p_{i}^{1}, q_{j}^{1} \in\left[p_{i}, q_{j}\right]$ is $p_{0}$ and $q_{0}$. This fact can be used to generate the points $p_{i}, 0,1, \ldots, I$, and $q_{j}, j=0,1, \ldots, J$. Although it is possible to determine these points directly from the definition, it is hard to derive a computationally efficient method to generate the points from inside to outside, i.e., from $p_{0}, q_{0}$ to $p_{I}, q_{J}$. In the following, we provide an iterative method based on the properties in Lemmas 4.1 and 4.2 to generate the points from outside to inside, i.e., from $p_{I}, q_{J}$ to $p_{0}, q_{0}$ and use the unique property that $p_{0}^{1}, q_{0}^{1} \in\left[p_{0}, q_{0}\right]$ as a sign to stop the iteration.

Algorithm for Generating $p_{i}, q_{j}, m_{i}, n_{j}$ and $p_{i}^{1}, q_{j}^{1}$

Step 1 Set $i i=1$. Get the two intersections of $A L_{h}^{e}$ with $\pm L_{h}^{e}$. They are $p_{I}^{1}$ and $-q_{J}^{1}$ (or $-p_{I}^{1}$ and $q_{J}^{1}$ ). Denote the line segment of $A L_{h}^{e}$ between $L_{h}^{e}$ and $-L_{h}^{e}$ as $L_{1}$. Multiply the two end points of $L_{1}$ from left with $A^{-1}$, then we get $p_{I}$ and $q_{J}$. Clearly, the one to the left of $p_{c}$ is $p_{I}$ and the one to its right is $q_{J}$. If $p_{I}^{1}, q_{J}^{1} \in\left[p_{I}, q_{J}\right]$, then $I=J=0$ and stop the algorithm.

Step $2 i i=i i+1$. Check if $A L_{i i-1}$ has intersections with $\pm L_{h}^{e}$. If not, let $L_{i i}=A L_{i i-1}$ and repeat this step. If there is, then cut off the part of $A L_{i i-1}$ that is outside of $\mathbf{S}^{e}$ and let the remaining part be $L_{i i}$. The cut-off place is one of $\pm p_{i}^{1}$ and $\pm q_{j}^{1}$. Multiply the cut-off place from left with $A^{-i i}$. The result is so far the innermost $p_{i}$ if it is to the left of $p_{c}$, or the innermost $q_{j}$ if to the right of $p_{c}$. In the mean time, we also obtain $m_{i}=i i$ and $\backslash$ or $n_{j}=i i$. Let the innermost pair be $p_{i}, q_{j}$, if $p_{i}^{1}, q_{j}^{1} \in\left[p_{i}, q_{j}\right]$, then we must have $i=j=0$ and stop the algorithm since all the $p_{i}, q_{j}$ have been computed. If $p_{i}^{1}, q_{j}^{1} \in\left[p_{i}, q_{j}\right]$ is not true, then repeat this step.

We see from the above algorithm that the number of iterations equals $\max \left\{m_{0}, n_{0}\right\}$.

Item 4) in Lemma 4.2 shows that $\left[p_{i-1}, p_{i-1}^{1}\right] \subset\left[p_{i}, p_{i}^{1}\right]$ and $\left[q_{j-1}^{1}, q_{j-1}\right] \subset\left[q_{j}^{1}, q_{j}\right]$. Item 5) shows that either we have $\left[p_{i}, p_{i}^{1}\right] \subset\left[q_{j}^{1}, q_{j}\right]$, or $\left[q_{j}^{1}, q_{j}\right] \subset\left[p_{i}, p_{i}^{1}\right]$. Item 3 ) shows that all these intervals must include $\left[p_{0}, q_{0}\right]$. In summary, the facts in Lemma 4.2 jointly show that the intervals $\left[p_{i}, p_{i}^{1}\right]$ and $\left[q_{j}^{1}, q_{j}\right]$, $i=0,1, \ldots, I, j=0,1, \ldots J$ are ordered by inclusion. We can draw a figure for easy understanding of Lemma 4.2. If we draw arcs from $p_{i}$ to $p_{i}^{1}$ and arcs from $q_{j}$ to $q_{j}^{1}$, then these arcs 


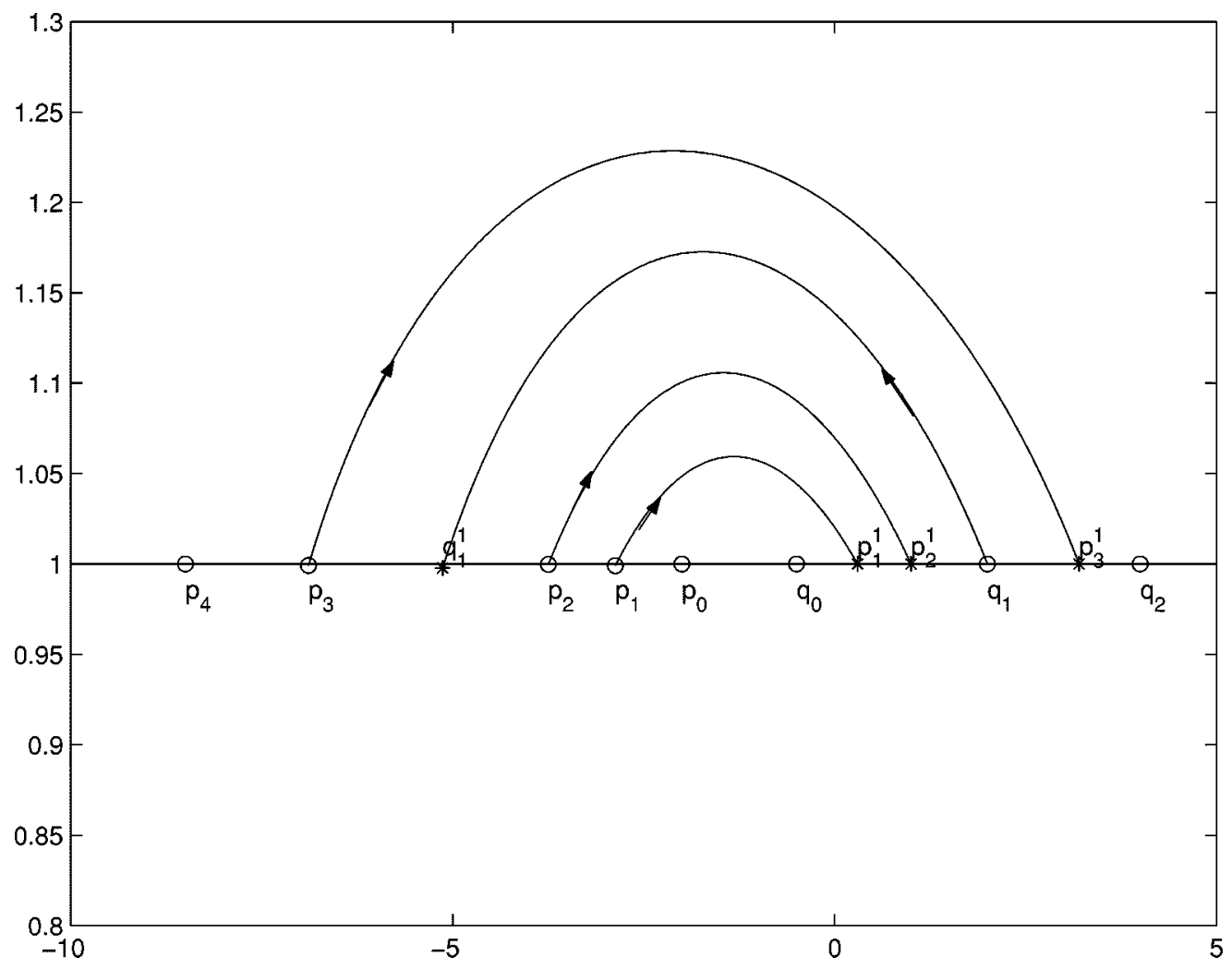

Fig. 2. Illustration for Lemma 4.2.

can be made not to intersect each other (see Fig. 2). In the figure, we have

\section{$\left[p_{0}, q_{0}\right] \subset\left[p_{1}, p_{1}^{1}\right] \subset\left[p_{2}, p_{2}^{1}\right] \subset\left[q_{1}^{1}, q_{1}\right] \subset\left[p_{3}, p_{3}^{1}\right]$.}

Let $p_{i}^{2}=\psi_{2}\left(m_{i-1}, p_{i}\right)=\operatorname{sat}_{2}\left(A^{m_{i-1}} p_{i}\right)$, then $p_{i}^{2}$ is the third intersection of $\psi_{2}\left(k, p_{i}\right)$ with $\pm L_{h}^{e}$. By Lemma 4.14$)$, we know that $m_{i-1}$ is the smallest integer $k$ such that $A^{k} p_{i}$ is outside of $\mathbf{S}^{e}$. Also let $q_{j}^{2}=\psi_{2}\left(n_{j-1}, q_{j}\right)=\operatorname{sat}_{2}\left(A^{n_{j-1}} q_{j}\right)$.

Lemma 4.3: Suppose that (9) is not true for any $x_{1} \in \mathbf{R}$, then $p_{i}^{2} \in\left(p_{i}, p_{i}^{1}\right)$ and $q_{j}^{2} \in\left(q_{j}^{1}, q_{j}\right)$.

Proof: Suppose $p_{i}^{1} \in\left(q_{j}, q_{j+1}\right]$, then by Lemma 4.22$)$, the smallest $k$ for $A^{k} p_{i}$ to go out of $\mathbf{S}^{e}$ is $k=m_{i-1}=m_{i}+n_{j}$. So $p_{i}^{2}=\operatorname{sat}_{2}\left(A^{m_{i}+n_{j}} p_{i}\right)=\operatorname{sat}_{2}\left(A^{n_{j}} p_{i}^{1}\right)$. If on the contrary that $p_{i}^{2} \in\left[p_{i}^{1}, q_{\infty}\right)$ (to the right of $p_{i}^{1}$ ), since $q_{j}^{1}=A^{n_{j}} q_{j} \in$ $\pm L_{h}^{e}$ is to the left of $q_{j}$, there must be a point $x \in\left(q_{j}, p_{i}^{1}\right) \subset$ $\left(q_{j}, q_{j+1}\right]$ such that $A^{n_{j}} x$ is right above $x$, i.e., $\operatorname{sat}_{2}\left(A^{n_{j}} x\right)=$ $x$. By Lemma 4.13), the next intersection of $\psi_{2}(k, x)$ with $\pm L_{h}^{e}$ is $\operatorname{sat}_{2}\left(A^{n_{j}} x\right)$, so we must have $A^{k} x \in \mathbf{S}^{e}$ for all $k<n_{j}$ and there exists $x_{1}$ such that (9) is true. A contradiction. On the other hand, if $p_{i}^{2}=\operatorname{sat}_{2}\left(A^{m_{i-1}} p_{i}\right) \in\left(p_{\infty}, p_{i}\right]$ (to the left of $p_{i}$ ), since $p_{i-1}^{1}=A^{m_{i-1}} p_{i-1}$ is to the right of $p_{i-1}$, there must be a point $x \in\left[p_{i}, p_{i-1}\right)$ such that $A^{m_{i-1}} x$ is right above $x$. Similar to the former case, we have a contradiction. Therefore, $p_{i}^{2} \in\left(p_{i}, p_{i}^{1}\right)$, and similarly, $q_{j}^{2} \in\left(q_{j}^{1}, q_{j}\right)$.

This lemma says that if a trajectory starts from $p_{i}$ or $q_{j}$, its third intersection with $\pm L_{h}^{e}$ will be closer to the central interval $\left[p_{0}, q_{0}\right]$ than the first intersection or the second one. We will show in the next lemma that this property can be actually extended to all $x \in L_{h}^{e}$.
Lemma 4.4: Assume that the condition (9) is not true for any $x_{1} \in \mathbf{R}$. Given $x(0)=x_{0} \in L_{h}^{e}$. Let $x_{0}^{1}$ and $x_{0}^{2}$ be the second and the third intersection of the trajectory $\psi_{2}\left(k, x_{0}\right)$ with $L_{h}^{e}$, (if the intersections are on $-L_{h}^{e}$, then get symmetric projections on $\left.L_{h}^{e}\right)$. If $x_{0} \in\left(p_{\infty}, p_{0}\right]$, then $x_{0}^{1} \in\left(x_{0}, q_{\infty}\right)$ and one of the following must be true.

1) $x_{0}^{1} \in\left(p_{0}, q_{0}\right)$ and there is no third intersection $x_{0}^{2}$;

2) $x_{0}^{1} \in\left(x_{0}, p_{0}\right]$

3) $x_{0}^{1} \in\left[q_{0}, q_{\infty}\right)$ and $x_{0}^{2} \in\left(x_{0}, x_{0}^{1}\right)$.

Similarly, if $x_{0} \in\left[q_{0}, q_{\infty}\right)$, then $x_{0}^{1} \in\left(p_{\infty}, x_{0}\right)$ and one of the following must be true.

4) $x_{0}^{1} \in\left(p_{0}, q_{0}\right)$ and there is no third intersection $x_{0}^{2}$;

5) $x_{0}^{1} \in\left[q_{0}, x_{0}\right)$;

6) $x_{0}^{1} \in\left(p_{\infty}, p_{0}\right]$ and $x_{0}^{2} \in\left(x_{0}^{1}, x_{0}\right)$.

Also, if $x_{0} \in\left(p_{i}, p_{i}^{1}\right)$ [or $x_{0} \in\left(q_{j}^{1}, q_{j}\right)$ ], then $x_{0}^{1}, x_{0}^{2}$ and the subsequent intersections will all be in the interval $\left(p_{i}, p_{i}^{1}\right)$ [or $\left.\left(q_{j}^{1}, q_{j}\right)\right]$. Furthermore, for any $x_{0} \in L_{h}^{e}$, there is a finite $k_{1}$ such that $\psi_{2}\left(k_{1}, x_{0}\right) \in\left(p_{0}, q_{0}\right)$. After that, $\psi_{2}\left(k, x_{0}\right)$ will have no more intersection with $L_{h}^{e}$ and will converge to the origin.

Proof: Lemma 4.2 says that all the segments $\left[p_{i}, p_{i}^{1}\right]$ and $\left[q_{j}^{1}, q_{j}\right]$ are ordered by inclusion. We will prove the result of this lemma from the innermost segment to the outermost with an inductive procedure. Without loss of generality, assume that $\left[p_{1}, p_{1}^{1}\right]$ is the innermost segment (except for $\left.\left[p_{0}, q_{0}\right]\right)$, then we must have $p_{1}^{1} \in\left(q_{0}, q_{1}\right]$ and $p_{1}^{2}=\operatorname{sat}_{2}\left(A^{n_{0}} p_{1}^{1}\right)=\operatorname{sat}_{2}\left(A^{m_{0}} p_{1}\right)$.

By Lemma 4.3, $p_{1}^{2} \in\left(p_{1}, p_{1}^{1}\right)$. There are three possibilities.

Case $1-p_{1}^{2} \in\left[q_{0}, p_{1}^{1}\right):$ (See Fig. 3.) For $x_{0} \in\left[q_{0}, p_{1}^{1}\right)$, $x_{0}^{1}=\operatorname{sat}_{2}\left(A^{n_{0}} x_{0}\right)$ by Lemma 4.13). Since $q_{0}^{1}=\operatorname{sat}_{2}\left(A^{n_{0}} q_{0}\right)$ and $p_{1}^{2}=\operatorname{sat}\left(A^{n_{0}} p_{1}^{1}\right)$ are to the left of $q_{0}$ and $p_{1}^{1}$ respectively, 


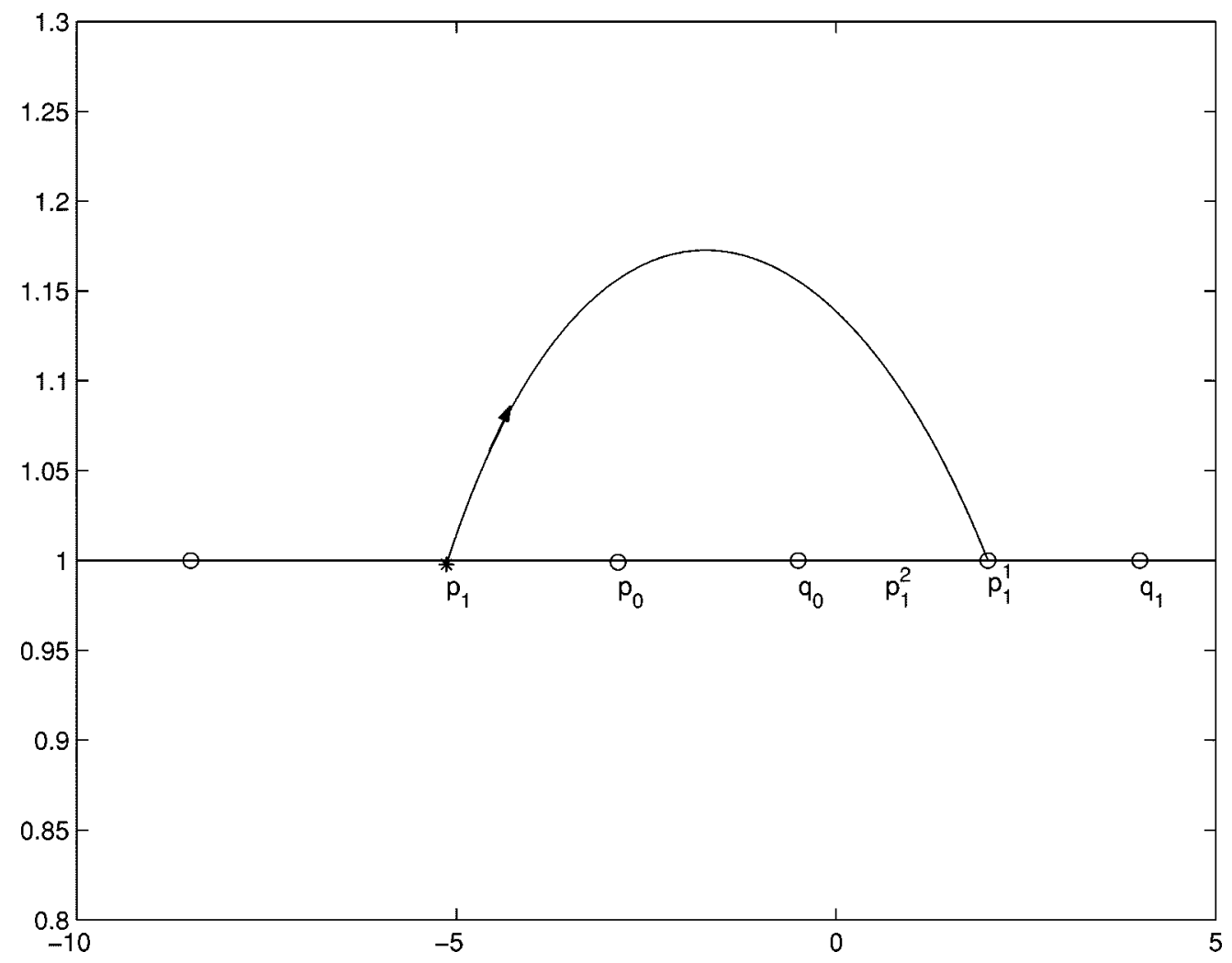

Fig. 3. Illustration for the proof of Lemma 4.4: Case 1.

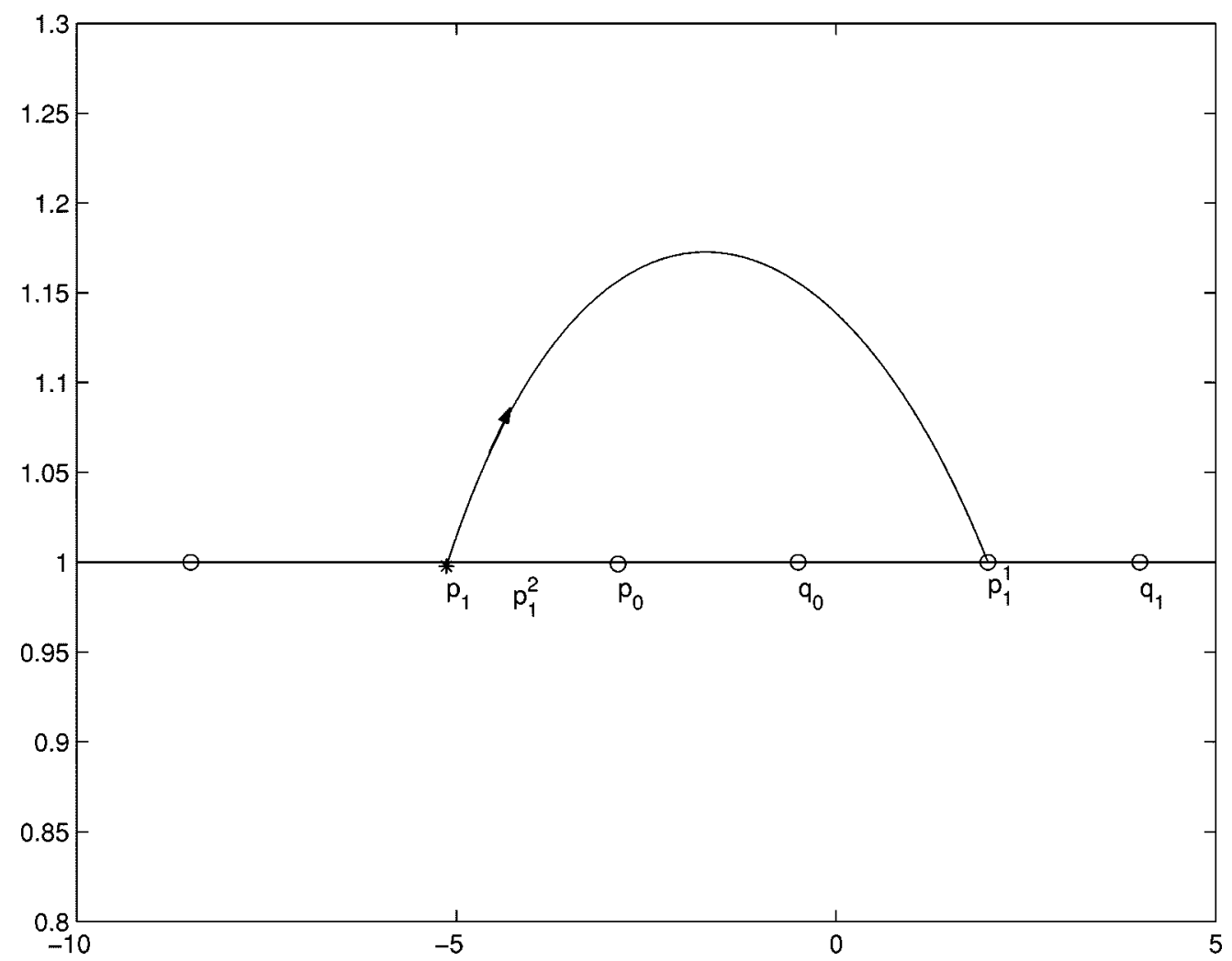

Fig. 4. Illustration for the proof of Lemma 4.4: Case 2.

$x_{0}^{1}$ must be to the left of $x_{0}$ and also, $x_{0}^{1} \in\left[q_{0}^{1}, p_{1}^{2}\right)$. So we have $x_{1}^{1} \in\left(q_{0}^{1}, x_{0}\right)$. This belongs to 4$)$ or 5$)$ of the lemma. If it is 4), then there will be no more intersection; If it is 5), then with the same argument, we have $x_{0}^{2} \in\left(q_{0}^{1}, x_{0}^{1}\right), \ldots$. Moreover, the subsequent intersections will fall between $p_{0}$ and $q_{0}$ in a finite number of steps since there is no $x_{1}$ satisfying (9). 


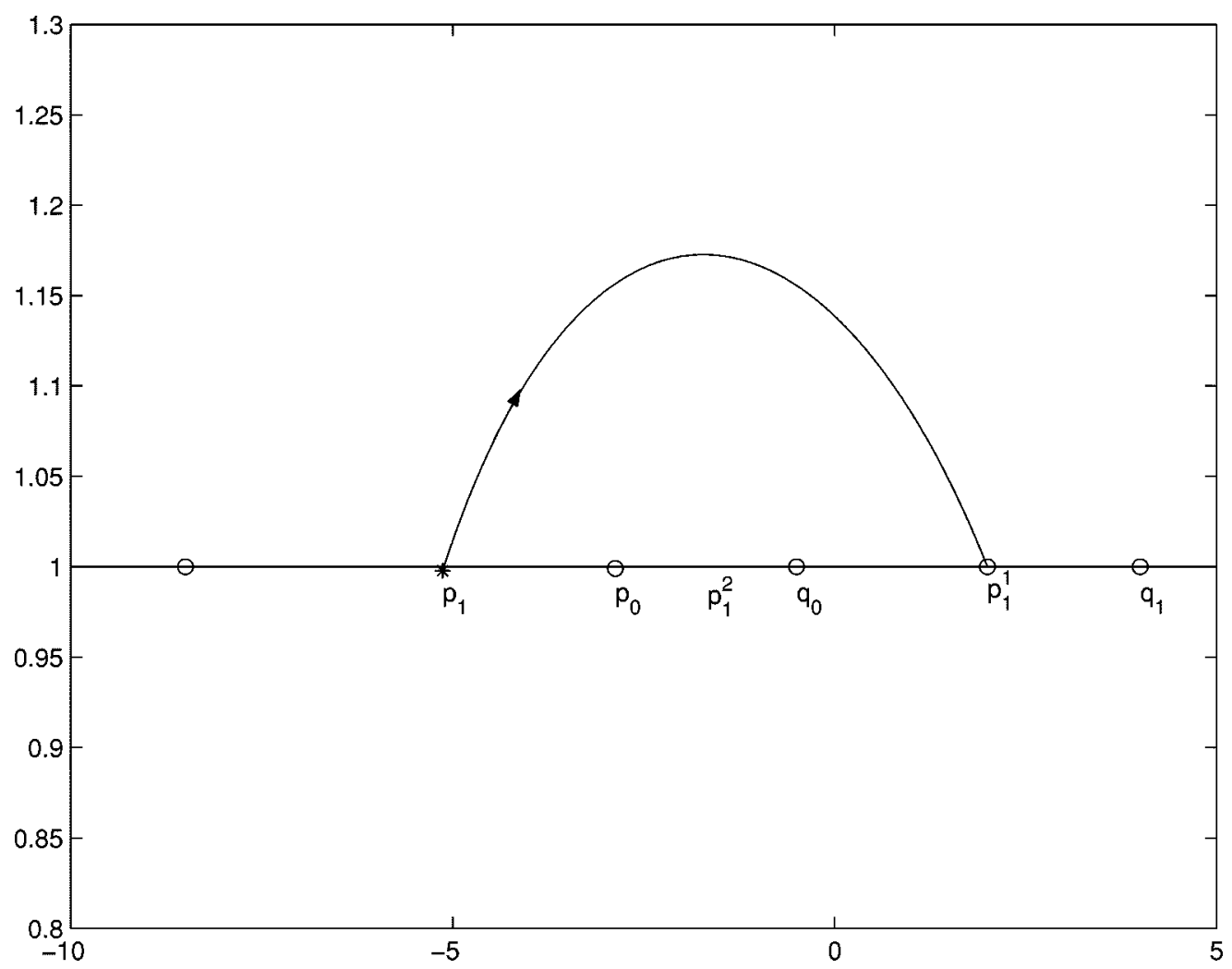

Fig. 5. Illustration for the proof of Lemma 4.4: Case 3.

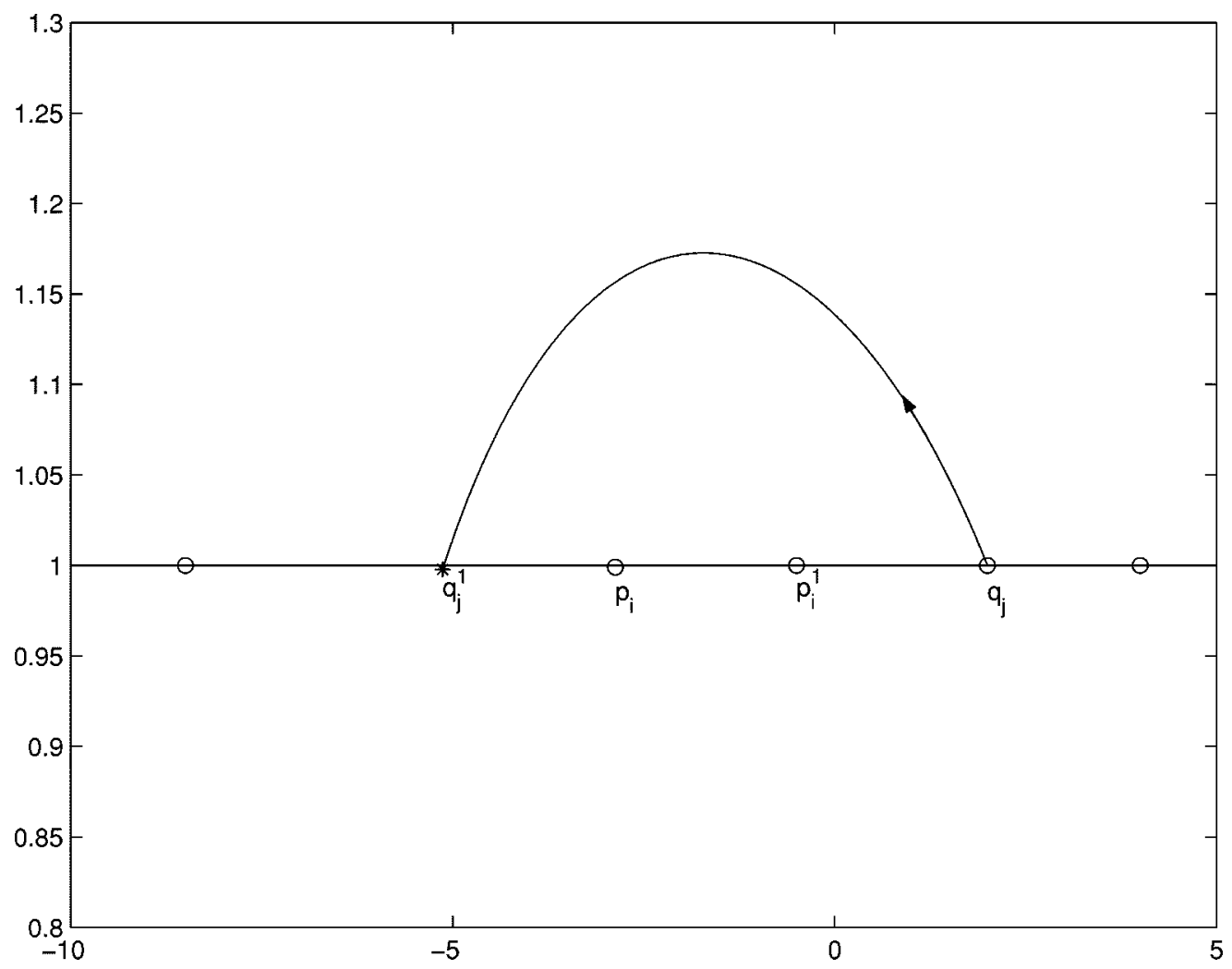

Fig. 6. Illustration for the proof of Lemma 4.4.

For $x_{0} \in\left(p_{1}, p_{0}\right], x_{0}^{1}=\operatorname{sat}_{2}\left(A^{m_{0}} x_{0}\right) \in\left[p_{0}^{1}, p_{1}^{2}\right)$. If $x_{0}^{1} \in$ $\left(p_{0}, q_{0}\right)$, then we get 1$)$ of the lemma. If $x_{0}^{1} \in\left[q_{0}, p_{1}^{2}\right)$, then by the argument in the previous paragraph, we must have $x_{0}^{2} \in$ $\left[q_{0}^{1}, x_{0}^{1}\right) \subset\left(x_{0}, x_{0}^{1}\right)$ and we get 3$)$ of the lemma. 


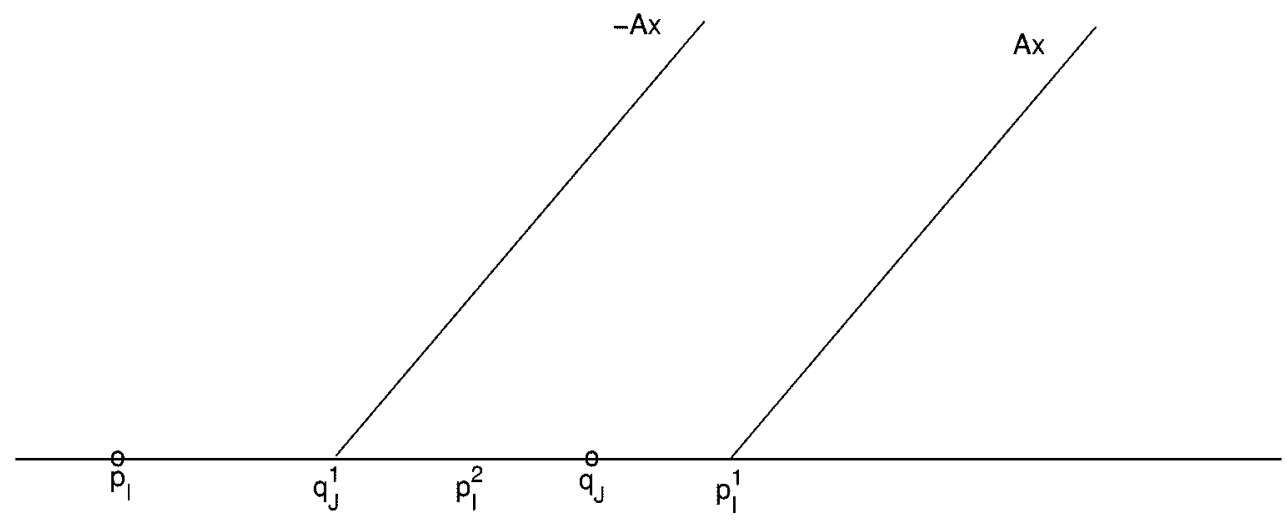

Fig. 7. Illustration for the proof of Lemma 4.4: Case i.

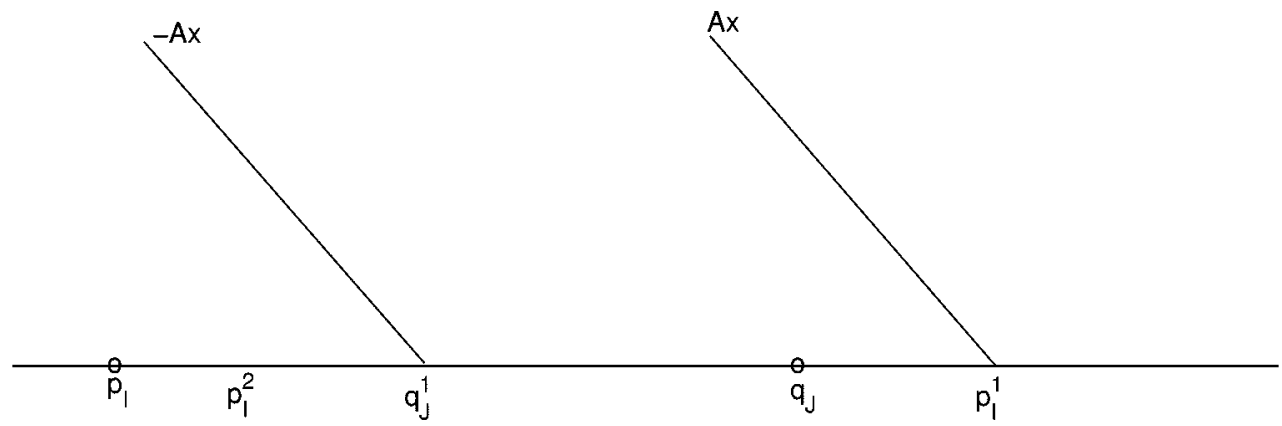

Fig. 8. Illustration for the proof of Lemma 4.4: Case ii.

Case $2-p_{1}^{2} \in\left(p_{1}, p_{0}\right]:$ (See Fig. 4.) For $x_{0} \in\left(p_{1}, p_{0}\right]$, $x_{0}^{1}=\operatorname{sat}_{2}\left(A^{m_{0}} x_{0}\right) \in\left(p_{1}^{2}, p_{0}^{1}\right]$. Since $p_{1}^{2}$ and $p_{0}^{1}$ are to the right of $p_{1}$ and $p_{0}$ respectively, $x_{0}^{1}$ must also be to the right of $x_{0}$, i.e., $x_{0}^{1} \in$ $\left(x_{0}, p_{0}^{1}\right]$. If $x_{0}^{1} \in\left(x_{0}, p_{0}\right)$, then we get 2$)$ and the subsequent intersections, if any, will moverightward untilfalling between $p_{0}$ and $q_{0}$; if $x_{0}^{1} \in\left(p_{0}, p_{0}^{1}\right) \subset\left(p_{0}, q_{0}\right)$, then we get 1$)$.

For $x_{0} \in\left[q_{0}, p_{1}^{1}\right), x_{0}^{1}=\operatorname{sat}_{2}\left(A^{n_{0}} x_{0}\right) \in\left(p_{1}^{2}, q_{0}^{1}\right)$. If $x_{0}^{1} \in$ $\left[p_{0}, q_{0}^{1}\right) \subset\left(p_{0}, q_{0}\right)$ then we obtain 4$)$. If $x_{0}^{1} \in\left(p_{1}^{2}, p_{0}\right]$, then the argument in the foregoing paragraph applies and we have $x_{0}^{2} \in\left(x_{0}^{1}, p_{0}^{1}\right] \subset\left(x_{0}^{1}, x_{0}\right)$, which belongs to 6$)$.

Case 3- $p_{1}^{2} \in\left(p_{0}, q_{0}\right):$ (See Fig. 5.) For $x_{0} \in\left(p_{1}, p_{0}\right]$, we have $x_{0}^{1} \in\left(p_{1}^{2}, p_{0}^{1}\right] \subset\left(p_{0}, q_{0}\right)$, which belongs to 1$)$. For $x_{0} \in\left[q_{0}, p_{1}^{1}\right)$, we have $x_{0}^{1} \in\left(p_{1}^{2}, q_{0}^{1}\right] \subset\left(p_{0}, q_{0}\right)$, which belongs to 4$)$.

So far, we have shown that one of 1)-6) holds for all $x_{0} \in$ $\left[p_{1}, p_{1}^{1}\right]$. And in each of the above three cases, we see that for all $x_{0} \in\left(p_{1}, p_{1}^{1}\right), x_{0}^{1}, x_{0}^{2}$ and the subsequent intersections are all in $\left(p_{1}, p_{1}^{1}\right)$ and will fall between $p_{0}$ and $q_{0}$ in a finite number of steps.

Next, we assume that these properties hold for all $x_{0} \in\left[p_{i}, p_{i}^{1}\right]$ and the next segment which includes $\left[p_{i}, p_{i}^{1}\right]$ is $\left[q_{j}^{1}, q_{j}\right]$ (see Fig. 6). We also have three cases: $q_{j}^{2} \in\left[p_{i}^{1}, q_{j}\right), q_{j}^{2} \in\left(q_{j}^{1}, p_{i}\right]$ and $q_{j}^{2} \in\left(p_{i}, p_{i}^{1}\right)$. By treating the segment $\left[p_{i}, p_{i}^{1}\right]$ as $\left[p_{0}, q_{0}\right]$ in the proof for $\left[p_{1}, p_{1}^{1}\right]$, we can use the same argument to show that one of 1)-6) holds for all $x_{0} \in\left(q_{j}^{1}, p_{i}\right] \cup\left[p_{i}^{1},, q_{j}\right)$. Moreover, for all $x_{0} \in\left(q_{j}^{1}, p_{i}\right] \cup\left[p_{i}^{1},, q_{j}\right)$, the intersections will move toward $\left[p_{i}, p_{i}^{1}\right]$ and fall between $\left[p_{i}, p_{i}^{1}\right]$ in a finite number of steps.
Now, suppose that $\left[p_{I}, p_{I}^{1}\right]$ is the outermost segment. By induction, we have obtained the properties in the lemma for all $x_{0} \in\left[p_{I}, p_{I}^{1}\right]$ and we would like to extend the properties to the whole line $L_{h}^{e}$.

Recall that $m_{I}=n_{J}=1$, so $p_{I}^{1}=A p_{I}$ and $p_{I}^{2}=\operatorname{sat}_{2}\left(A^{2} p_{I}\right)=\operatorname{sat}_{2}\left(A p_{I}^{1}\right)$. The line $A L_{h}^{e}$ will actually intersect with $\pm L_{h}^{e}$ at $p_{I}^{1}$ and $-q_{J}^{1}$ (or $-p_{I}^{1}$ and $q_{J}^{1}$ ). Assume that $p_{I}^{1}$ is on $L_{h}^{e}$. Then the ray $\left\{A x: x \in\left[q_{J}, q_{\infty}\right)\right\}$ is below the line $-L_{h}^{e}$. Get a symmetric projection of this ray as $\left\{-A x: x \in\left[q_{J}, q_{\infty}\right)\right\}$. Then the two rays $\left\{-A x: x \in\left[q_{J}, q_{\infty}\right)\right\}$ and $\left\{A x: x \in\left(p_{\infty}, p_{I}\right]\right\}$ are parallel and are both above the line $L_{h}^{e}$ (see Figs. 7 and 8). Here, we have two cases.

Case $i$ : The rays have a positive slope (see Fig. 7). Since $p_{I}^{1} \in\left[q_{J}, q_{\infty}\right)$, we have $p_{I}^{2} \in\left[q_{J}^{1}, q_{\infty}\right)$ and by Lemma 4.3, $p_{I}^{2} \in\left(p_{I}, p_{I}^{1}\right)$. So, $p_{I}^{2} \in\left(q_{J}^{1}, p_{I}^{1}\right)$.

For $x_{0} \in\left(p_{I}^{1}, q_{\infty}\right)$, since both $q_{J}^{1}=-\operatorname{sat}_{2}\left(A q_{J}\right)$ and $p_{I}^{2}=$ - $\operatorname{sat}_{2}\left(A p_{I}^{1}\right)$ are to the left of $q_{J}$ and $p_{I}^{1}$, respectively, and since there exists no $x \in L_{h}^{e}$ such that $-\operatorname{sat}_{2}(A x)=x$, we must have, $x_{0}^{1}=-\operatorname{sat}_{2}\left(A x_{0}\right)$ to the left of $x_{0}$ and $x_{0}^{1} \in\left(p_{I}^{2}, x_{0}\right)$. If $x_{0}^{1} \in\left(p_{0}, q_{0}\right)$, then we obtain 4). If $x_{0}^{1} \in\left[q_{0}, x_{0}\right)$, then we obtain 5). If $x_{0}^{1} \in\left(q_{J}^{1}, p_{0}\right) \subset\left(q_{J}^{1}, q_{J}\right)$, then by the established properties on $\left[q_{J}^{1}, q_{J}\right]$, we must have $x_{0}^{2} \in\left(x_{0}^{1}, q_{J}\right)$ to the left of $p_{I}^{1}$ and hence to the left of $x_{0}$. Therefore, $x_{0}^{2} \in\left(x_{0}^{1}, x_{0}\right)$ and we get 6).

For $x_{0} \in\left(p_{\infty}, p_{I}\right), x_{0}^{1}=\operatorname{sat}_{2}(A x)$ is to the right of $p_{I}^{1}$, so the properties for $x_{0} \in\left(p_{I}^{1}, p_{\infty}\right)$ applies. Also note that $x_{0}^{2}$ is to the right of $p_{I}^{2}$. Hence $x_{0}^{2} \in\left(x_{0}, x_{0}^{1}\right)$ and we obtain 3$)$. 


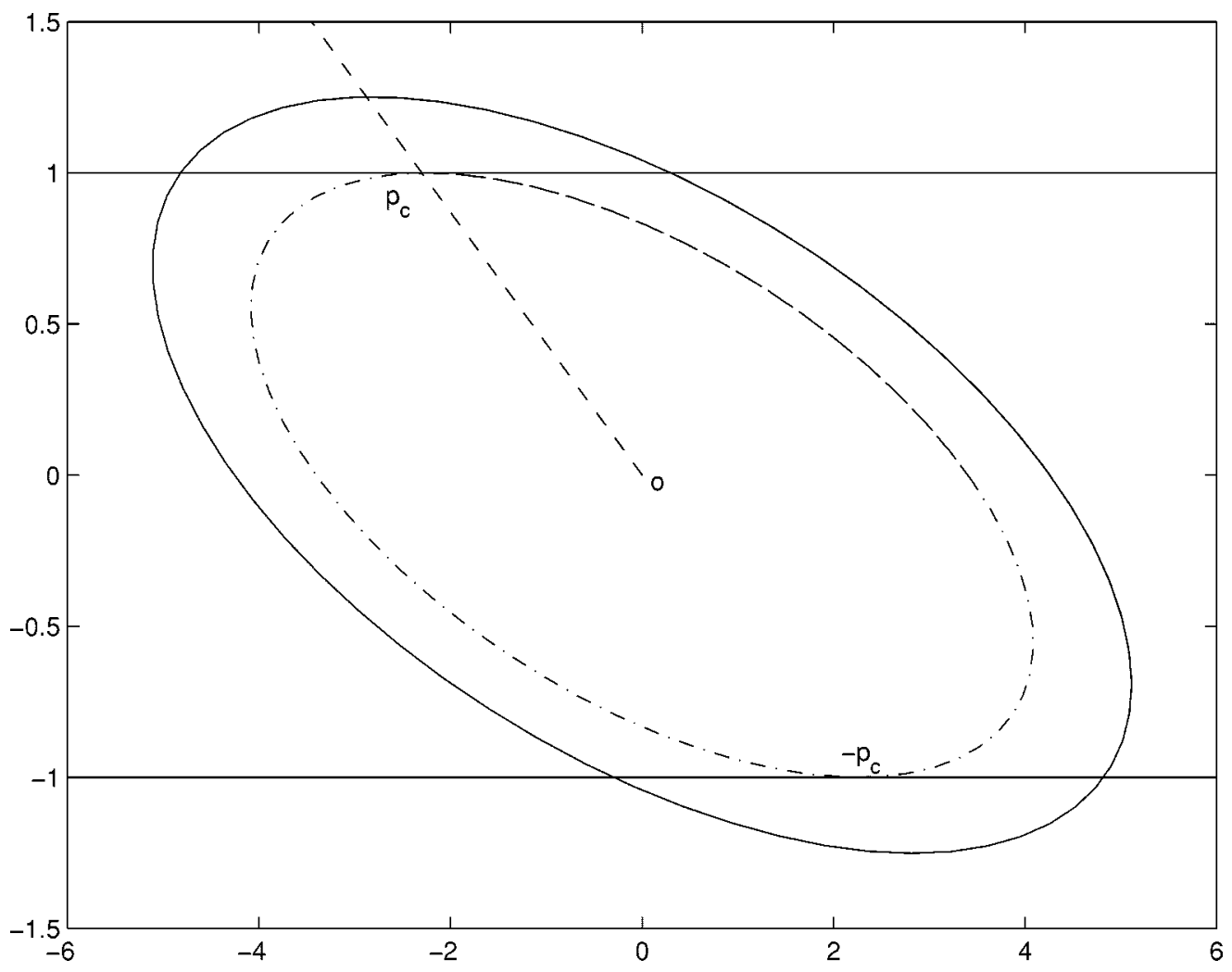

Fig. 9. Illustration for the proof of Lemma 4.5.

Case ii: The rays have a negative slope (see Fig. 8). In this case, $p_{I}^{2} \in\left(p_{I}, q_{J}^{1}\right)$.

For $x_{0} \in\left(p_{\infty}, p_{I}\right)$, since $p_{I}^{1}=A p_{I}$ is to the right of $p_{I}$ and there exist no $x \in L_{h}^{e}$ such that $\operatorname{sat}_{2}(A x)=x$, we must have $x_{0}^{1}=\operatorname{sat}_{2}\left(A x_{0}\right)$ to the right of $x_{0}$, in particular, $x_{0} \in\left(x_{0}, p_{I}^{1}\right)$. If $x_{0}^{1} \in\left(x_{0}, p_{0}\right]$, then we obtain 2). If $x_{0}^{1} \in\left(p_{0}, q_{0}\right)$, then we have 1$)$. If $x_{0}^{1} \in\left(q_{0}, p_{I}^{1}\right)$, then by using the established property in the interval $\left(p_{I}, p_{I}^{1}\right)$, we have $x_{0}^{2} \in\left(p_{I}, x_{0}^{1}\right) \subset\left(x_{0}, x_{0}^{1}\right)$ and we obtain 3$)$.

For $x_{0} \in\left(p_{I}^{1}, q_{\infty}\right), x_{0}^{1}=-\operatorname{sat}_{2}(A x)$ is to the right of $p_{I}^{2}$. By applying the property for $x_{0}$ in $\left(p_{\infty}, p_{I}\right)$ and $\left(p_{I}, p_{I}^{1}\right)$, we have $x_{0}^{2} \in\left(x_{0}^{1}, x_{0}\right)$, which belongs to $\left.\mathrm{f}\right)$.

Similar to the argument for the interval $\left(p_{1}, p_{1}^{1}\right)$, it can be shown that the intersections will fall between $\left(p_{I}, p_{I}^{1}\right)$ in a finite number of steps for all $x_{0} \notin\left(p_{I}, p_{I}^{1}\right)$.

In summary, the intersections of a trajectory $\psi_{2}\left(k, x_{0}\right)$ with the lines $\pm L_{h}^{e}$ will move from the outer intervals to the inner intervals until falling into $\left(p_{0}, q_{0}\right)$ in a finite number of steps. After that, it will not touch the lines $\pm L_{h}^{e}$ and will converge to the origin.

Next, we suppose that the condition (9) is true for some $x_{1} \in$ $\mathbf{R}$. We would like to determine an interval in $L_{h}^{e}$ such that a trajectory starting from this interval will converge to the origin.

Recall that $p_{c}$ is defined to be the unique intersection of the Lyapunov ellipsoid $\mathcal{E}\left(\rho_{c}\right)$ with the line $L_{h}^{e}$ (see Fig. 9). Also, $\alpha_{c}$ is the first coordinate of $p_{c}$, i.e., $p_{c}=\left[\begin{array}{c}\alpha_{c} \\ 1\end{array}\right]$.

Lemma 4.5: Assume that $\alpha_{c} \leq 0$. If there exist an integer $N>0$ and a $d>0$ such that $A^{N}\left[\begin{array}{c}x_{1} \\ 1\end{array}\right]= \pm\left[\begin{array}{c}x_{1} \\ 1+d\end{array}\right]$, then we must have $x_{1}<\alpha_{c}<0$.
Proof: When $\alpha_{c} \leq 0$, an ellipsoid $\mathcal{E}(\rho)$ takes the shape in Fig. 9. Each ellipsoid $\mathcal{E}(\rho)$ has an intersection with the ray that starts from the origin and passes through $p_{c}$. This intersection is the highest point in the ellipsoid. Since $\alpha_{c} \leq 0$, it can be seen that

$$
V\left(\left[\begin{array}{c}
x_{1} \\
1
\end{array}\right]\right)<V\left( \pm\left[\begin{array}{c}
x_{1} \\
1+d
\end{array}\right]\right) \quad \forall x_{1} \geq \alpha_{c}, \quad d>0 .
$$

Since

$$
V\left(A^{N}\left[\begin{array}{c}
x_{1} \\
1
\end{array}\right]\right)<V\left(\left[\begin{array}{c}
x_{1} \\
1
\end{array}\right]\right)
$$

it is impossible to have $A^{N}\left[\begin{array}{c}x_{1} \\ 1\end{array}\right]= \pm\left[\begin{array}{c}x_{1} \\ 1+d\end{array}\right]$ for any $x_{1} \geq \alpha_{c}$.

Lemma 4.6: Let

$$
\alpha_{s}=\min \left\{\left|x_{1}\right|: x_{1} \text { satisfies (9) }\right\} \text {. }
$$

Case 1) $\alpha_{c} \leq 0$. Let

$$
p_{s}=\left[\begin{array}{c}
-\alpha_{s} \\
1
\end{array}\right]
$$

then $p_{s}^{1}=p_{s} \in\left(p_{\infty}, p_{0}\right)$. Suppose that $p_{s} \in$ $\left[p_{i+1}, p_{i}\right)$. Then, for every $x_{0} \in\left(p_{s}, p_{i}^{1}\right]$, the trajectory $\psi_{2}\left(k, x_{0}\right)$ will converge to the origin;

Case 2) $\alpha_{c}>0$. Let

$$
p_{s}=\left[\begin{array}{c}
\alpha_{s} \\
1
\end{array}\right]
$$

then $p_{s}^{1}=p_{s} \in\left(q_{0}, q_{\infty}\right)$. Suppose that $p_{s} \in$ $\left(q_{j}, q_{j+1}\right]$. Then, for every $x_{0} \in\left[q_{j}^{1}, p_{s}\right]$, the trajectory $\psi_{2}\left(k, x_{0}\right)$ will converge to the origin. 
In both cases, no limit trajectory can be formed completely inside the strip

$$
\left\{\left[\begin{array}{l}
x_{1} \\
x_{2}
\end{array}\right]:\left|x_{1}\right|<\alpha_{s}\right\}
$$

Proof: We only prove Case 1 . Since $\alpha_{c} \leq 0$, by Lemma 4.5 , there is no $x_{1} \geq \alpha_{c}$ satisfying (9), so we have $-\alpha_{s}<$ $\alpha_{c}<0$ and $p_{s}$ must be between $p_{i+1}$ and $p_{i}$ for some $i$, noting that $p_{s}$ cannot be in $\left[p_{0}, q_{0}\right]$ by Lemma 4.11$)$. Following the iterative procedure in the proof of Lemma 4.4, we can show that for all $x_{0} \in\left[p_{i}, p_{i}^{1}\right]$, the trajectory will converge to the origin. Now we consider a point between $p_{s}$ and $p_{i}$. For $x_{0}$ in this interval, the next intersection of the trajectory with the lines $\pm L_{h}^{e}$ is $x_{0}^{1}=\operatorname{sat}_{2}\left(A^{m_{i}} x_{0}\right)$. Since $\operatorname{sat}_{2}\left(A^{m_{i}} p_{s}\right)=p_{s}\left(\right.$ or $\left.-p_{s}\right)$ and $p_{i}^{1}=\operatorname{sat}_{2}\left(A^{m_{i}} p_{i}\right)$ is to the right of $p_{i}$, we must have $x_{0}^{1} \in$ $\left(x_{0}, p_{i}^{1}\right)$, and the subsequent intersections will move rightward and fall between $p_{i}$ and $p_{i}^{1}$ in a finite number of steps. Therefore, the trajectory $\psi_{2}\left(k, x_{0}\right)$ will converge to the origin.

Now, consider $x_{0} \in\left(p_{i}^{1}, q_{\infty}\right) \subset\left(p_{c}, q_{\infty}\right)$. Let $k_{1}$ be the minimal integer such that $A^{k_{1}} x_{0}$ goes out of $\mathbf{S}^{e}$, then by the shape of the Lyapunov ellipsoid, the point $A^{k_{1}} x_{0}$ must be to the left of $x_{0}$ (or to the right of $-x_{0}$ if $A^{k} x_{0}$ is below the line $\left.-L_{h}^{e}\right)$, otherwise we would have $V\left(A^{k_{1}} x_{0}\right)>V\left(x_{0}\right)$, which is impossible. Hence, $x_{0}^{1}=\operatorname{sat}_{2}\left(A^{k_{1}} x_{0}\right)$ must be to the left of $x_{0}$, and the subsequent intersections either fall between $p_{s}$ and $p_{i}^{1}$ at a finite step, or go to the left of $p_{s}$. This shows that no limit trajectory can be formed completely to the right of $p_{s}$ and symmetrically, to the left of $-p_{s}$. Hence, no limit trajectory can be formed completely inside the strip

$$
\left\{\left[\begin{array}{l}
x_{1} \\
x_{2}
\end{array}\right]:\left|x_{1}\right|<\alpha_{s}\right\}
$$

Proof of Proposition 4.1: It follows immediately from Lemmas 4.4 and 4.6.

The number $\alpha_{s}$ and the point $p_{s}=\left[\begin{array}{c} \pm \alpha_{s} \\ 1\end{array}\right]$ can be easily computed by applying Lemma 4.1. Actually, all the $x_{1}$ satisfying (9) can be determined. Let $x_{0}=\left[\begin{array}{c}x_{1} \\ 1\end{array}\right]$, then $x_{1}$ satisfies (9) for some $N$ if and only if $x_{0}$ satisfies

$$
A^{N} x_{0} \notin \mathbf{S}^{e} \quad A^{k} x_{0} \in \mathbf{S}^{e} \quad \forall k<N
$$

and

$$
\operatorname{sat}_{2}\left(A^{N} x_{0}\right)=x_{0} .
$$

Assume that $\alpha_{c}<0$, then by Lemma 4.5, we only need to check if there is such an $x_{0}$ in the interval $\left(p_{\infty}, p_{0}\right)$. Clearly, no $x_{0}$ in $\left[p_{0}, q_{0}\right]$ satisfies (12) by Lemma 4.11 ). So we need to check over the intervals $\left[p_{i+1}, p_{i}\right)$ with $i$ increased from 0 to $I-1$ and the interval $\left(p_{\infty}, p_{I}\right)$.

Consider a point $x_{0}$ in the interval $\left[p_{i+1}, p_{i}\right)$. By Lemma 4.1 2), the smallest integer $N$ for $A^{N} x_{0} \notin \mathbf{S}^{e}$ is $N=m_{i}$. By Lemma 4.2 3), $A^{m_{i}} p_{i}=p_{i}^{1} \in\left(q_{0}, q_{\infty}\right)$ is to the right of $p_{i}$. So there exists $x_{0} \in\left[p_{i+1}, p_{i}\right)$ satisfying (12) if and only if $A^{m_{i}} p_{i+1}$ is to the left of $p_{i+1}$, i.e.,

$$
\operatorname{sat}_{2}\left(A^{m_{i}} p_{i+1}\right) \in\left(p_{\infty}, p_{i+1}\right] .
$$

If this is true, then $x_{1}$, the first coordinate of $x_{0}$, can be solved from

$$
\left[\begin{array}{ll}
1 & 0
\end{array}\right] A^{m_{i}}\left[\begin{array}{c}
x_{1} \\
1
\end{array}\right]= \pm x_{1} .
$$

In summary, we have the following

Algorithm for Determining All the $x_{1}$ Satisfying Condition (9): Assume $\alpha_{c} \leq 0$. Initially set $i=0$.

Step 1) $i=i+1$. If (13) is satisfied, then compute $x_{1}$ from (14). Repeat this step until $i=I-1$.

Step 2) Solve

$$
\left[\begin{array}{ll}
1 & 0
\end{array}\right] A\left[\begin{array}{c}
x_{1} \\
1
\end{array}\right]= \pm x_{1}
$$

for $x_{1}$, if $x_{1} \in\left(-\infty, \alpha_{I}\right)$, then $x_{1}$ satisfies (9) with $N=1$.

\section{Proof of the MaIn Results}

Now, we turn back to the system (5),

$$
x(k+1)=\operatorname{sat}(A x(k)) .
$$

For easy reference, we restate Theorem 2.1 as follows.

Theorem 5.1: The system (15) is globally asymptotically stable if and only if $A$ is stable and none of the following statements are true.

1) There exists an $N \geq 1$ such that

$$
\left.\operatorname{sat}\left(A^{N} v_{1}\right)\right)= \pm v_{1} \quad \text { and } \quad A^{k} v_{1} \in \mathbf{S} \quad \forall k<N .
$$

2) There exists an $N \geq 1$ such that

$$
\left.\operatorname{sat}\left(A^{N} v_{2}\right)\right)= \pm v_{2} \quad \text { and } \quad A^{k} v_{2} \in \mathbf{S} \quad \forall k<N .
$$

3) There exists an $x_{1} \in(-1,1)$ and an $N \geq 1$ such that

and

$$
\operatorname{sat}\left(A^{N}\left[\begin{array}{c}
x_{1} \\
1
\end{array}\right]\right)= \pm\left[\begin{array}{c}
x_{1} \\
1
\end{array}\right]
$$

$$
A^{k}\left[\begin{array}{c}
x_{1} \\
1
\end{array}\right] \in \mathbf{S} \quad \forall k<N .
$$

4) There exists an $x_{2} \in(-1,1)$ and an $N \geq 1$ such that

$$
\operatorname{sat}\left(A^{N}\left[\begin{array}{c}
1 \\
x_{2}
\end{array}\right]\right)= \pm\left[\begin{array}{c}
1 \\
x_{2}
\end{array}\right]
$$

and

$$
A^{k}\left[\begin{array}{c}
1 \\
x_{2}
\end{array}\right] \in \mathbf{S} \quad \forall k<N .
$$

Proof: We will exclude the possibility of the existence of limit trajectories (except for the trivial one at the origin) under the condition that none of statements 1)-4) in the theorem is true. In the following, when we say a limit trajectory, we mean a nontrivial one other than the origin. Clearly, every limit trajectory must include at least one point on the boundary of the unit square, i.e., a point in the set $\pm\left(L_{h} \cup L_{v} \cup\left\{v_{1}, v_{2}\right\}\right)$. By Proposition 3.1, we know that a limit trajectory cannot have points in both $\pm L_{h}$ and $\pm L_{v}$. So we have two possibilities here, limit trajectories including points in $\pm\left(L_{h} \cup\left\{v_{1}, v_{2}\right\}\right)$, and those including points in $\pm\left(L_{v} \cup\left\{v_{1}, v_{2}\right\}\right)$. Because of the similarity, 
we only exclude the first possibility under the condition that none of 1)-3) is true, the second possibility can be excluded under the condition that none of 1),2) and 4) is true.

For a given initial state $x_{0}$, we denote the trajectory of the system (15) as $\psi\left(k, x_{0}\right)$ and the trajectory of (7) as $\psi_{2}\left(k, x_{0}\right)$.

Clearly, if $x_{1} \in(-1,1)$ satisfies 3$)$, then this $x_{1}$ also satisfies (9). On the other hand, suppose that there is some $x_{1}$ that satisfies (9). Let $p_{s}$ be as defined in Lemma 4.6 for the system (7) [if there is no $x_{1}$ that satisfies (9), then we can assume that

$$
p_{s}=\left[\begin{array}{c} 
\pm \infty \\
1
\end{array}\right]
$$

and the following argument also goes through]. Note that, if there is some $x_{1} \in \mathbf{R},\left|x_{1}\right| \leq 1$, that satisfies (9), i.e.,

$$
A^{N}\left[\begin{array}{c}
x_{1} \\
1
\end{array}\right]= \pm\left[\begin{array}{c}
x_{1} \\
1+d
\end{array}\right]
$$

and

$$
\left|\left[\begin{array}{ll}
0 & 1
\end{array}\right] A^{k}\left[\begin{array}{c}
x_{1} \\
1
\end{array}\right]\right| \leq 1 \quad \forall k<N
$$

we must also have

$$
\left|\left[\begin{array}{ll}
1 & 0
\end{array}\right] A^{k}\left[\begin{array}{c}
x_{1} \\
1
\end{array}\right]\right| \leq\left|x_{1}\right| \quad \forall k<N
$$

which indicates that $x_{1}$ satisfies 3 ). Otherwise, as in the proof of Proposition 3.1, the area of the convex hull of the set

$$
\left\{ \pm x_{0}, \pm A x_{0}, \ldots, \pm A^{N-1} x_{0}\right\}
$$

would be less than the area of the convex hull of the set

$$
\left\{ \pm A x_{0}, \pm A^{2} x_{0}, \ldots, \pm A^{N} x_{0}\right\} .
$$

This would be a contradiction to the fact that $|\operatorname{det}(A)|<1$.

Hence, if no $x_{1}$ satisfies 3 ), then $p_{s}$ must be outside of $\mathbf{S}$. By Proposition 4.1, no limit trajectory of (7) can lie completely inside the strip

$$
\left\{\left[\begin{array}{l}
x_{1} \\
x_{2}
\end{array}\right]:\left|x_{1}\right|<\alpha_{s}\right\} .
$$

It follows that no limit trajectory of (15) can lie completely between $-L_{v}$ and $L_{v}$. Therefore, no limit trajectory of (15) can include only boundary points in $\pm L_{h}$. On the other hand, if a limit trajectory include only boundary points $\pm v_{1}$ (or $\pm v_{2}$, note that, by Proposition 3.1, no limit trajectory can include both $\pm v_{1}$ and $\pm v_{2}$ ), then 1) or 2) must be true, which contradicts our assumption. In short, if there is a limit trajectory that include points in $\pm\left(L_{h} \cup\left\{v_{1}, v_{2}\right\}\right)$, it must include at least one point on $\pm L_{h}$ and one on $\pm v_{1}$ (or $\pm v_{2}$ ). Here, we assume that it includes $v_{2}$.

Let us consider the trajectories $\psi\left(k, v_{2}\right)$ and $\psi_{2}\left(k, v_{2}\right)$. Suppose that $\psi\left(k, v_{2}\right)$ has an intersection with $\pm L_{h}$ but does not include $v_{1}$ and any point in $\pm L_{v}$, we conclude that $\psi\left(k, v_{2}\right)=$ $\psi_{2}\left(k, v_{2}\right)$ will converge to the origin. The argument goes as follows.

Let $k_{0}$ be the smallest $k$ such that $\psi\left(k, v_{2}\right)$ intersects $\pm L_{h}$. Denote $v_{2}^{1}=\psi\left(k_{0}, v_{2}\right)$. Since 2) is not true, $k_{0}$ must also be the smallest $k$ such that

$$
\left|\left[\begin{array}{ll}
0 & 1
\end{array}\right] A^{k} v_{2}\right| \geq 1
$$

So, we have $\psi_{2}\left(k, v_{2}\right)=\psi\left(k, v_{2}\right)$ for all $k \leq k_{0}$. Here, we have two cases.

Case $1-\alpha_{c} \leq 0$ : In this case, $p_{s}$ is to the left of $v_{2}$. Since $v_{2}^{1}=\operatorname{sat}\left(A^{k_{0}} v_{2}\right)=\operatorname{sat}_{2}\left(A^{k_{0}} v_{2}\right)$ goes to the right of $v_{2}$, by Lemma $4.5, v_{2}$ must be to the left of $p_{0}$. It follows that $v_{2} \in$ $\left(p_{s}, p_{i}^{1}\right]$, where $\left(p_{s}, p_{i}^{1}\right]$ is the interval in Lemma 4.62$)$. Hence, $\psi_{2}\left(k, v_{2}\right)$ will converge to the origin. Moreover, the subsequent intersections of $\psi_{2}\left(k, v_{2}\right)$ with $\pm L_{h}$ are between $v_{2}$ and $v_{2}^{1}$. Since $\psi\left(k, v_{2}\right)$ does not touch $\pm L_{v}$, we must have $\psi\left(k, v_{2}\right)=$ $\psi_{2}\left(k, v_{2}\right)$ and hence $\psi\left(k, v_{2}\right)$ will also converge to the origin.

Case $2-\alpha_{c}>0$ : In this case $p_{s}$ is to the right of $v_{1}$. By the assumption that $\psi\left(k, v_{2}\right)$ does not include $v_{1}$, the intersections of $\psi_{2}\left(k, v_{2}\right)$ with $\pm L_{h}$ will stay to the left of $v_{1}$ (or to the right of $-v_{1}$ ). Since $\alpha_{c}>0$, by Lemma 4.5, the intersections will move rightward until falling on $\left[q_{j}^{1}, p_{s}\right)$, where $\left[q_{j}^{1}, p_{s}\right)$ is the interval in Lemma 4.63 ). Similar to Case 1, we have that $\psi_{2}\left(k, v_{2}\right)$ converges to the origin and $\psi\left(k, v_{2}\right)=\psi_{2}\left(k, v_{2}\right)$.

So far, we have excluded the possibility that a limit trajectory includes any point in the set $\pm\left(L_{h} \cup\left\{v_{1}, v_{2}\right\}\right)$. The possibility that a limit trajectory includes any point in the set $\pm\left(L_{v} \cup\right.$ $\left.\left\{v_{1}, v_{2}\right\}\right)$ can be excluded in a similar way. Thus, there exists no limit trajectory of any kind and the system (15) must be globally asymptotically stable.

Here we provide a simple method to check the conditions 3) and 4) of Theorem 5.1 based on the algorithm to determine all the $x_{1}$ satisfying (9) and hence $p_{s}$ in the previous section. From the proof of Theorem 5.1, we see that 3) is true if and only if $p_{s} \in \mathbf{S}$. To check 4), we can exchange $x_{1}$ and $x_{2}$, i.e., use a state transformation $y=\left[\begin{array}{ll}0 & 1 \\ 1 & 0\end{array}\right] x$. The system (15) is then equivalent to

$$
y(k+1)=\operatorname{sat}(\bar{A} y(k))
$$

where $\bar{A}=\left[\begin{array}{ll}0 & 1 \\ 1 & 0\end{array}\right] A\left[\begin{array}{ll}0 & 1 \\ 1 & 0\end{array}\right]$. The condition 4) for the system (15) is equivalent to the condition 3 ) for the system (16).

\section{CONCLUSIONS}

We gave a complete stability analysis of a planar discrete-time linear system under saturation. The analysis involves intricate investigation on the intersections of the trajectories with the lines $x_{1}= \pm 1$ and $x_{2}= \pm 1$. Our main result provides a necessary and sufficient condition for such a system to be globally asymptotically stable.

\section{REFERENCES}

[1] F. Albertini and D. D'Alessandro, "Asymptotic stability of continuous-time systems with saturation nonlinearities," Syst. Control Lett., vol. 29, no. 3, pp. 175-180, 1996.

[2] D. S. Bernstein and A. N. Michel, "A chronological bibliography on saturating actuators," Int. J. Robust Nonlinear Control, vol. 5, no. 5, pp. $375-380,1995$.

[3] L. Hou and A. N. Michel, "Asymptotic stability of systems with saturation constraints," IEEE Trans. Automat. Contr., vol. 43, pp. 1148-1154, Aug. 1998.

[4] T. Hu and Z. Lin, "A complete stability analysis of planar linear systems under saturation," IEEE Trans. Circuits Syst. I, vol. 47, pp. 498-512, Apr. 2000.

[5] L. Jin, P. N. Nikiforuk, and M. M. Gupta, "Absolute stability conditions for discrete-time recurrent neural networks," IEEE Trans. Neural Networks, vol. 5, pp. 954-964, Nov. 1994.

[6] Z. Lin, Low Gain Feedback, ser. Lecture Notes in Control and Information Sciences. London, U.K.: Springer, 1998, vol. 240. 
[7] D. Liu and A. N. Michel, "Asymptotic stability of systems operating on a closed hypercube," Syst. Control Lett., vol. 19, no. 4, pp. 281-285, 1992.

[8] — "Sparsely interconnected neural networks for associative memories with applications to cellular neural networks," IEEE Trans. Circuits Syst., vol. 41, pp. 295-307, Apr. 1994.

[9] - Dynamical Systems with Saturation Nonlinearities, ser. Lecture Notes in Control and Information Sciences. London: Springer, 1994, vol. 195.

[10] R. Mantri, A. Saberi, and V. Venkatasubramanian, "Stability analysis of continuous time planar systems with state saturation nonlinearity," IEEE Trans. Circuits Syst. I, vol. 45, pp. 989-993, Sept. 1998.

[11] J. H. F. Ritzerfeld, "A condition for the overflow stability of secondorder digital filters that is satisfied by all scaled state-space structures using saturation," IEEE Trans. Circuits Syst., vol. 36, pp. 1049-1057, Aug. 1989.

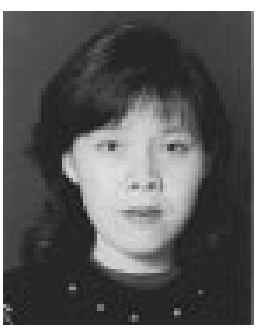

Tingshu Hu was born in Sichuan, China in 1966 She received the B.S. and M.S. degrees in electrical engineering from Shanghai Jiao Tong University, Shanghai, China, in 1985 and 1988, respectively. She is currently working toward the Ph.D degree in electrical engineering, at the University of Virginia, Charlottesville.

Her research interests include systems with saturation nonlinearities and robust control theory, areas in which she has published several papers. She is also the co-author (with Zongli Lin) of the book Contro Systems with Actuator Saturation: Analysis and Design (Birkhäuser, Boston, 2001).

Ms. Hu is an Associate Editor on the Conference Editorial Board of the IEEE Control Systems Society.

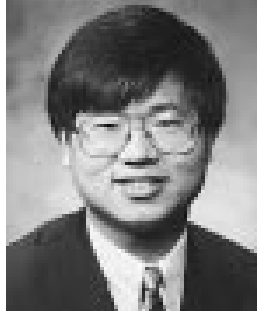

Zongli Lin (S'89-M'90-SM'98) was born in Fuqing, Fujian, China, in 1964. He received the B.S. degree in mathematics and computer science from Amoy University, Xiamen, China, in 1983, the Master of Engineering degree in automatic control from the Chinese Academy of Space Technology, Beijing, China, in 1989, and the Ph.D. degree in electrical and computer engineering, from Washington State University, Pullman, WA, in May 1994.

From July 1983 to July 1986, Dr. Lin worked as a Control Engineer at Chinese Academy of Space Technology. In January 1994, he joined the Department of Applied Mathematics and Statistics, State University of New York, Stoney Brook as a Visiting Assistant Professor, where he became an Assistant Professor in September 1994. Since July 1997, he has been an Assistant Professor in electrical engineering at University of Virginia, Charlottesville. His current research interests include nonlinear control, robust control, and control of systems with saturating actuators. In these areas he has published several papers. He is also the author of the recent book, Low Gain Feedback (Springer-Verlag, London, 1998) and a co-author (with Tingshu Hu) of the book Control Systems with Actuator Saturation: Analysis and Design (Birkhäuser, Boston, 2001).

Dr. Lin has been an Associate Editor on the Conference Editorial Board of the IEEE Control Systems Society and currently serves as an Associate Editor of IEEE TRANSACTIONS ON AUTOMATIC CONTROL. He is the recipient of a U.S. Office of Naval Research Young Investigator Award. 